\title{
Adaptive processing for beamforming with coherent interference using uniform circular arrays
}

\author{
Ju-Hong Lee*, Shuo-Wei Hsiao \\ Department of Electrical Engineering, Graduate Institute of Communication Engineering and Graduate Institute of Biomedical Electronics \\ and Bioinformatics, National Taiwan University, Roosevelt Rd., Taipei 10617, Taiwan
}

Available online 13 November 2007

\begin{abstract}
The paper deals with the problem of adaptive array beamforming using a uniform circular array (UCA) in the presence of coherent interference. The well-known scheme of spatial smoothing (SS) widely used to tackle the coherent problem in the cases of 1-D uniform linear array (ULA) and uniform rectangular array (URA) cannot work for the UCA case. This is mainly due to the fact that the direction vector of each signal received by a UCA does not possess a Vandermonde structure. In this paper, we present a method based on subarray beamforming in conjunction with an averaging scheme to effectively mitigate the effect due to coherent interference in the UCA case. Theoretical analysis regarding the validity of the proposed method is also presented. Several simulation examples are provided for showing the effectiveness of the proposed method.
\end{abstract}

(c) 2007 Elsevier Inc. All rights reserved.

Keywords: Adaptive beamforming; Uniform circular array (UCA); Coherent signal sources

\section{Introduction}

An adaptive array beamformer is designed for automatically preserving the desired signals while canceling interference and noise. The explosive growth of wireless communications stimulates the research efforts investigating the design and analysis of 2-D adaptive beamformers using a uniform circular array (UCA) [1-8]. A 2-D array beamformer can discriminate the signals overlapping spectrally and temporally from multiple mobile users. This ability makes smart antennas very suitable for wireless communication systems in several aspects like capacity enhancement, quality improvement in the presence of coherent or multipath signal sources, and the possibility to increase the throughput at the network nodes that is limited by interferences from neighboring nodes. Several typical scenarios, such as radar, sonar, and cellular phones, etc., involve all-azimuth angle coverage in the plane of the array without significant change in either the beam width or the sidelobe level [1]. Among 2-D adaptive beamformers, the beamformer with a UCA structure has been widely used because the UCA structure possesses symmetry and provides an appropriate solution to scenarios wherein $360^{\circ}$ field-of-view is required [2].

In the case without coherent interference, an optimum UCA beamformer works successfully for desired signal reception and interference rejection. However, it has been shown in [3] that the performance of a UCA beamformer

\footnotetext{
* Corresponding author. Fax: +886223671909.

E-mail address: juhong@cc.ee.ntu.edu.tw (J.-H. Lee).
} 


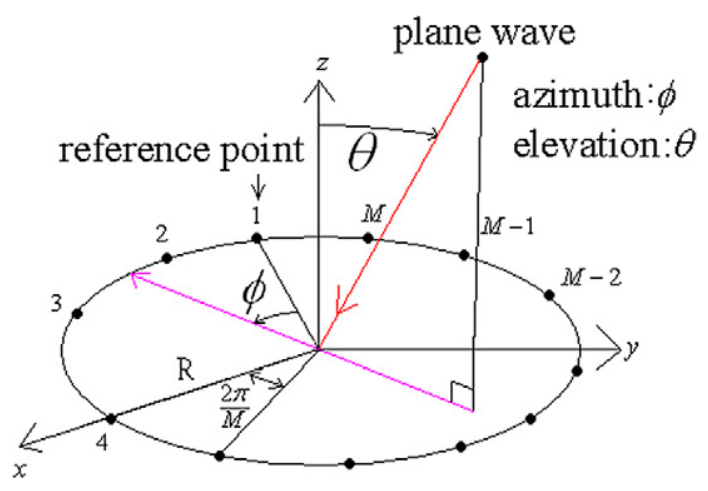

Fig. 1. The geometric illustration of a uniform circular array.

suffers from severe degradation in the presence of correlation between desired signal and interference, e.g., in a multipath environment. To cure the problem of array performance degradation due to correlated signal sources, many techniques [3,9-15] suggest to employ a transformation of the data format received by the actual array to a data format received by a virtual array whose structure is suitable for using the conventional spatial smoothing (SS) scheme [16]. Due to that the steering vector of a transformed virtual array demonstrates the desired Vandermonde structure, the SS scheme can therefore exploit the Vandermonde property to destroy the correlation between signal sources and counteract the signal cancellation phenomenon. However, the proposed transformation scheme in general introduces an approximation and results in an approximate Vandermonde form for the steering vector of the resultant virtual array. As a result, some compromises in terms of the size of the virtual array are required. In contrast, the CSMA technique of [17] employs a rotating circular array. The incident signal at each array element is sampled within an angular region where the curvature of the circular array is assumed to be linear. The coherent signals can be decorrelated by taking the average of the covariance matrix during a certain rotating time interval. Although the technique can alleviate the effect due to signal coherence without using the above transformation, some conditions related to the UCA structure must be satisfied in order to ensure the resulting covariance matrix is nonsingular.

In this paper, we consider the problem of adaptive beamforming using a UCA under the coherent interference. A method based on subarray beamforming [18-20] in conjunction with an averaging scheme to effectively mitigate the effect due to coherent signals is presented. A UCA is partitioned into overlapped subarrays with size $L$. Each of the resulting subarrays is configured in a circular arc possessing the same radius as the original UCA. The data correlation matrix of each subarray is computed and used for finding the optimal weight vector for the subarray. The interference at the output of each subarray can then be suppressed considerably if the subarray size $L$ is chosen appropriately. Using the outputs of the subarrays, we carry out an adaptive virtual beamforming which enhances the performance of the UCA. An efficient approach is presented to estimate the correlation matrix of the noise vector to facilitate the virtual beamforming. The theoretical analysis showing the validity of the proposed method is presented. Several simulation examples provide the illustration and comparison.

This paper is organized as follows. Section 2 formulates the problem of adaptive beamforming using UCA under coherent interference. Section 3 presents a method for dealing with the considered problem. In Section 4, we present a theoretical analysis for showing the validity of the proposed method. Section 5 presents simulation results to show the effectiveness of the proposed method. Finally, we conclude the paper in Section 6.

\section{Problem formulation}

Consider a uniform circular array (UCA) with $M$ isotropic sensors located in the $X-Y$ plane as shown by Fig. 1. Let $R$ be the radius and $d$ be the spacing between two adjacent elements. Moreover, we set $d=(2 R) \sin (\pi / M)$. Assume that $K$ narrow-band and far-field signals are impinging on the array with angles $\left(\theta_{k}, \phi_{k}\right), k=1,2, \ldots, K$, where $\theta_{k}$ and $\phi_{k}$ represent the elevation and azimuth angles, respectively. The direction vector of the UCA with respect to the center of the array for the $k$ th signal with angel $\left(\theta_{k}, \phi_{k}\right)$ and wavelength $\lambda$ is given by

$$
\boldsymbol{a}\left(\theta_{k}, \phi_{k}\right)=\left[a_{1}\left(\theta_{k}, \phi_{k}\right) a_{2}\left(\theta_{k}, \phi_{k}\right) \ldots a_{M}\left(\theta_{k}, \phi_{k}\right)\right]^{\mathrm{T}},
$$


where $a_{m}\left(\theta_{k}, \phi_{k}\right)$ denotes the phase delay at the $m$ th array sensor and is given by

$$
a_{m}\left(\theta_{k}, \phi_{k}\right)=\exp \left\{j 2 \pi \frac{R}{\lambda} \cos \left[\phi_{k}-\frac{2 \pi(m-1)}{M}\right] \sin \left(\theta_{k}\right)\right\}, \quad m=1,2, \ldots, M,
$$

where $j=\sqrt{-1}$. The signal received at the $m$ th array sensor can be expressed as

$$
x_{m}(t)=\sum_{i=1}^{K} s_{i}(t) a_{m}\left(\theta_{i}, \phi_{i}\right)+n_{m}(t), \quad m=1,2, \ldots, M,
$$

where $s_{i}(t)$ is the complex waveform of the $i$ th signal, and $n_{m}(t)$ is the spatially white noise with mean zero and variance $\sigma_{n}^{2}$ received at the $m$ th array sensor. In matrix form, we can write the data vector received by the UCA as follows:

$$
\boldsymbol{x}(t)=\boldsymbol{A s}(t)+\boldsymbol{n}(t),
$$

where the matrix $\boldsymbol{A}=\left[\boldsymbol{a}\left(\theta_{1}, \phi_{1}\right) \boldsymbol{a}\left(\theta_{2}, \phi_{2}\right) \ldots \boldsymbol{a}\left(\theta_{K}, \phi_{K}\right)\right]$ with the direction vector of the $k$ th signal given by (1), the signal source vector is $\boldsymbol{s}(t)=\left[\begin{array}{llll}s_{1}(t) & s_{2}(t) & \ldots & s_{K}(t)\end{array}\right]^{\mathrm{T}}$, and the noise vector is $\boldsymbol{n}(t)=\left[\begin{array}{lllll}n_{1}(t) & n_{2}(t) & \ldots & n_{M}(t)\end{array}\right]^{\mathrm{T}}$. The superscript $\mathrm{T}$ denotes transpose operation. Assume that $\boldsymbol{s}(t)$ and $\boldsymbol{n}(t)$ are uncorrelated, the $M \times M$ ensemble correlation matrix of $\boldsymbol{x}(t)$ is given by

$$
\boldsymbol{R}_{x}=E\left\{\boldsymbol{x}(t) \boldsymbol{x}(t)^{\mathrm{H}}\right\}=\boldsymbol{A} \boldsymbol{R}_{s} \boldsymbol{A}^{\mathrm{H}}+\sigma_{n}^{2} \boldsymbol{I}_{M},
$$

where the superscript $\mathrm{H}$ denotes complex conjugate transpose, $\boldsymbol{R}_{s}=E\left\{\boldsymbol{s}(t) \boldsymbol{s}(t)^{\mathrm{H}}\right\}$ denotes the signal correlation matrix and has rank $K$ if the $K$ signal sources are uncorrelated, and $\boldsymbol{I}_{M}$ is the identity matrix with size $M \times M$.

Let the UCA use a weight vector $\boldsymbol{w}=\left[\begin{array}{llll}w_{1} & w_{2} & \ldots & w_{M}\end{array}\right]^{\mathrm{T}}$ for processing the received data vector $\boldsymbol{x}(t)$ and the array output be $y(t)=\boldsymbol{w}^{\mathrm{H}} \boldsymbol{x}(t)$. Assume that the selective signal requirements are specified by assigning a gain $c_{p}\left(0 \leqslant c_{p} \leqslant 1\right)$ at the direction vector $\boldsymbol{a}\left(\theta_{p}, \phi_{p}\right)$ for $p=1,2, \ldots, P$, where $P$ is the number of the specified signals with gain constraint. Then, the optimal weight vector can be found from the following constrained optimization problem:

$$
\text { minimize } E\left\{|y(t)|^{2}\right\}=\boldsymbol{w}^{\mathrm{H}} \boldsymbol{R}_{x} \boldsymbol{w}, \quad \text { subject to } \boldsymbol{G}^{\mathrm{H}} \boldsymbol{w}=\boldsymbol{c},
$$

where the matrix $\boldsymbol{G}=\left[\boldsymbol{a}\left(\theta_{1}, \phi_{1}\right) \boldsymbol{a}\left(\theta_{2}, \phi_{2}\right) \ldots \boldsymbol{a}\left(\theta_{P}, \phi_{P}\right)\right]$ denotes the constraint matrix and $\boldsymbol{c}=\left[\begin{array}{llll}c_{1} & c_{2} & \ldots & c_{P}\end{array}\right]^{\mathrm{T}}$ the corresponding gain vector. The optimal weight vector for (6) is given by

$$
\boldsymbol{w}=\boldsymbol{R}_{x}^{-1} \boldsymbol{G}\left(\boldsymbol{G}^{\mathrm{H}} \boldsymbol{R}_{x}^{-1} \boldsymbol{G}\right)^{-1} \boldsymbol{c} .
$$

Substituting (7) into $E\left\{|y(t)|^{2}\right\}=w^{\mathrm{H}} \boldsymbol{R}_{x} w$ yields the array output power as follows:

$$
E\left\{|y(t)|^{2}\right\}=\boldsymbol{w}^{\mathrm{H}} \boldsymbol{R}_{x} \boldsymbol{w}=\boldsymbol{c}^{\mathrm{H}}\left(\boldsymbol{G}^{\mathrm{H}} \boldsymbol{R}_{x}^{-1} \boldsymbol{G}\right)^{-1} \boldsymbol{c} .
$$

The beamformer formulated by (6) is referred to as the conventional UCA beamformer. In the presence of coherent interference, the performance of the UCA beamformers will be deteriorated because the eigenstructure of the correlation matrix $\boldsymbol{R}_{x}$ is destroyed. This is mainly due to the fact that the rank of $\boldsymbol{A} \boldsymbol{R}_{S} \boldsymbol{A}^{\mathrm{H}}$ is reduced by the coherence between signal sources.

\section{Proposed method}

Here, we present a subarray processing method to deal with the problem of coherent signals. Research works on subarray beamforming with some advantages over conventional beamforming using a ULA have been represented in [18-20]. Fig. 2 shows the UCA beamformer with size $L$ for each subarray and $L-1$ array sensors overlapped for two adjacent subarrays.

\subsection{Subarray beamformer}

Let the UCA be partitioned into $N$ subarrays. Each of the resulting subarrays is configured in an $L$-element circular arc possessing the same radius as the original UCA. Let the number of array sensors overlapped for two adjacent subarrays be $L-u$. The data vector received by the $l$ th subarray is given by

$$
\boldsymbol{x}_{l}(t)=\left[\begin{array}{llll}
x_{l 1}(t) & x_{l 2}(t) \ldots x_{l L}(t)
\end{array}\right]^{\mathrm{T}}
$$




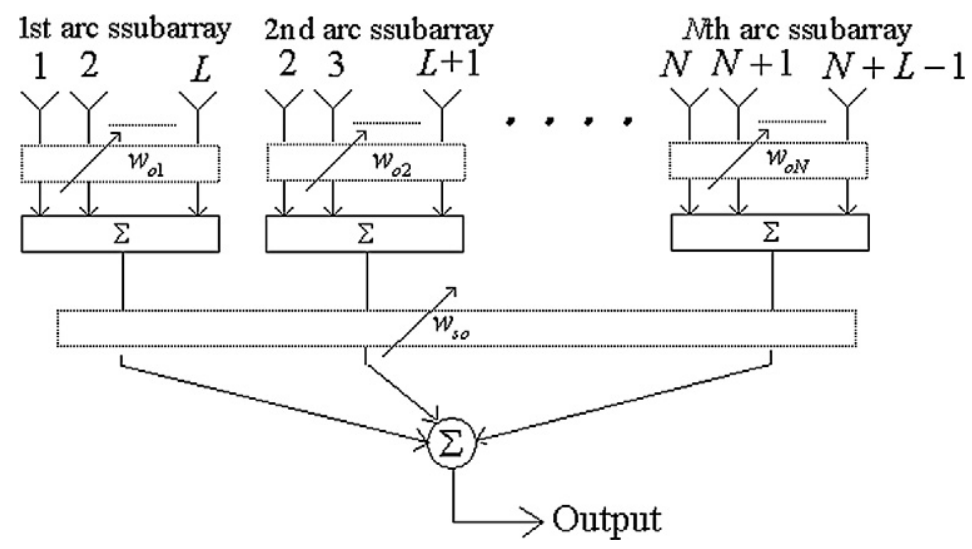

Fig. 2. The configuration of a UCA subarray beamforming.

where $x_{l q}(t)=x_{(l-1) n+q}(t)$ denotes the $q$ th element of $\boldsymbol{x}_{l}(t)$. Next, we compute the optimal weight vector for the $l$ th subarray, $l=1,2, \ldots, N$. From (3), (4), and (9), the data vector received by the $l$ th subarray and the corresponding $L \times L$ ensemble correlation matrix can be expressed as

$$
\boldsymbol{x}_{l}(t)=\boldsymbol{A}_{l} \boldsymbol{s}(t)+\boldsymbol{n}_{l}(t), \quad \boldsymbol{R}_{l}=E\left\{\boldsymbol{x}_{l}(t) \boldsymbol{x}_{l}(t)^{\mathrm{H}}\right\}=\boldsymbol{A}_{l} \boldsymbol{R}_{s} A_{l}^{\mathrm{H}}+\sigma_{n}^{2} \boldsymbol{I}_{L},
$$

respectively, where $\boldsymbol{A}_{l}=\left[\boldsymbol{a}_{l}\left(\theta_{1}, \phi_{1}\right) \boldsymbol{a}_{l}\left(\theta_{2}, \phi_{2}\right) \ldots \boldsymbol{a}_{l}\left(\theta_{K}, \phi_{K}\right)\right]$ with the direction vector of the $i$ th signal given by $\boldsymbol{a}_{l}\left(\theta_{i}, \phi_{i}\right)=\left[\begin{array}{llll}a_{(l-1) n+1}\left(\theta_{i}, \phi_{i}\right) & a_{(l-1) n+2}\left(\theta_{i}, \phi_{i}\right) & \ldots & a_{(l-1) n+L}\left(\theta_{i}, \phi_{i}\right)\end{array}\right]^{\mathrm{T}}$, the signal source vector is $\boldsymbol{s}(t)=$ $\left[s_{1}(t) s_{2}(t) \ldots s_{K}(t)\right]^{\mathrm{T}}$, and the noise vector is $\boldsymbol{n}_{l}(t)=\left[\begin{array}{llll}n_{(l-1) n+1}(t) & n_{(l-1) n+2}(t) \ldots n_{(l-1) n+L}(t)\end{array}\right]^{\mathrm{T}}$. Following (6) and (7), the optimal weight vector for the $l$ th subarray is given by

$$
\boldsymbol{w}_{o l}=\boldsymbol{R}_{l}^{-1} \boldsymbol{G}_{l}\left(\boldsymbol{G}_{l}^{\mathrm{H}} \boldsymbol{R}_{l}^{-1} \boldsymbol{G}_{l}\right)^{-1} \boldsymbol{c},
$$

where $\boldsymbol{G}_{l}=\left[\boldsymbol{a}_{l}\left(\theta_{1}, \phi_{1}\right) \boldsymbol{a}_{l}\left(\theta_{2}, \phi_{2}\right) \ldots \boldsymbol{a}_{l}\left(\theta_{P}, \phi_{P}\right)\right]$ denotes the constraint matrix for the $l$ th subarray. The output of the $l$ th subarray is given by

$$
y_{l}(t)=\boldsymbol{w}_{o l}^{\mathrm{H}} \boldsymbol{x}_{l}(t), \quad l=1,2, \ldots, N .
$$

The vector $\boldsymbol{y}(t)=\left[\begin{array}{lllll}y_{1}(t) & y_{2}(t) & \ldots & y_{N}(t)\end{array}\right]^{\mathrm{T}}$ containing the outputs of the subarrays is expressed by

$$
\begin{aligned}
\boldsymbol{y}(t) & =\sum_{p=1}^{P}\left[\begin{array}{c}
\boldsymbol{w}_{o 1}^{\mathrm{H}} \boldsymbol{a}_{1}\left(\theta_{p}, \phi_{p}\right) \\
\boldsymbol{w}_{o 2}^{\mathrm{H}} \boldsymbol{a}_{2}\left(\theta_{p}, \phi_{p}\right) \\
\vdots \\
\boldsymbol{w}_{o N}^{\mathrm{H}} \boldsymbol{a}_{N}\left(\theta_{p}, \phi_{p}\right)
\end{array}\right] s_{p}(t)+\sum_{p=P+1}^{K}\left[\begin{array}{c}
\boldsymbol{w}_{o 1}^{\mathrm{H}} \boldsymbol{a}_{1}\left(\theta_{p}, \phi_{p}\right) \\
\boldsymbol{w}_{o 2}^{\mathrm{H}} \boldsymbol{a}_{2}\left(\theta_{p}, \phi_{p}\right) \\
\vdots \\
\boldsymbol{w}_{o N}^{\mathrm{H}} \boldsymbol{a}_{N}\left(\theta_{p}, \phi_{p}\right)
\end{array}\right] s_{p}(t)+\left[\begin{array}{c}
\boldsymbol{w}_{o 1}^{\mathrm{H}} \boldsymbol{n}_{1}(t) \\
\boldsymbol{w}_{o 2}^{\mathrm{H}} \boldsymbol{n}_{2}(t) \\
\vdots \\
\boldsymbol{w}_{o N}^{\mathrm{H}} \boldsymbol{n}_{N}(t)
\end{array}\right] \\
& =\boldsymbol{y}_{s}(t)+\boldsymbol{y}_{i}(t)+\boldsymbol{y}_{n}(t),
\end{aligned}
$$

where $\boldsymbol{y}_{s}(t), \boldsymbol{y}_{i}(t)$, and $\boldsymbol{y}_{n}(t)$ represent the desired signal component, the interference component, and the noise component of $\boldsymbol{y}(t)$, respectively. Since there are $L-u$ array sensors overlapped between two adjacent subarrays, the noises in $\boldsymbol{y}_{n}(t)$ of (13) are no longer spatially white.

\subsection{Virtual beamforming}

From (13), the $N \times N$ ensemble correlation matrices of $\boldsymbol{y}_{s}(t), \boldsymbol{y}_{j}(t)$, and $\boldsymbol{y}_{n}(t)$ are given by

$$
\boldsymbol{R}_{s 2}=E\left\{\boldsymbol{y}_{s}(t) \boldsymbol{y}_{s}(t)^{\mathrm{H}}\right\}=\sum_{p=1}^{P} E\left\{\left|s_{p}(t)\right|^{2}\right\}\left[\begin{array}{c}
\boldsymbol{w}_{o 1}^{\mathrm{H}} \boldsymbol{a}_{1}\left(\theta_{p}, \phi_{p}\right) \\
\boldsymbol{w}_{o 2}^{\mathrm{H}} \boldsymbol{a}_{2}\left(\theta_{p}, \phi_{p}\right) \\
\vdots \\
\boldsymbol{w}_{o N}^{\mathrm{H}} \boldsymbol{a}_{N}\left(\theta_{p}, \phi_{p}\right)
\end{array}\right]\left[\begin{array}{c}
\boldsymbol{w}_{o 1}^{\mathrm{H}} \boldsymbol{a}_{1}\left(\theta_{p}, \phi_{p}\right) \\
\boldsymbol{w}_{o 2}^{\mathrm{H}} \boldsymbol{a}_{2}\left(\theta_{p}, \phi_{p}\right) \\
\vdots \\
\boldsymbol{w}_{o N}^{\mathrm{H}} \boldsymbol{a}_{N}\left(\theta_{p}, \phi_{p}\right)
\end{array}\right]^{\mathrm{H}},
$$




$$
\boldsymbol{R}_{j 2}=E\left\{\boldsymbol{y}_{j}(t) \boldsymbol{y}_{j}(t)^{\mathrm{H}}\right\}=\sum_{p=P+1}^{K} E\left\{\left|s_{p}(t)\right|^{2}\right\}\left[\begin{array}{c}
\boldsymbol{w}_{o 1}^{\mathrm{H}} \boldsymbol{a}_{1}\left(\theta_{p}, \phi_{p}\right) \\
\boldsymbol{w}_{o 2}^{\mathrm{H}} \boldsymbol{a}_{2}\left(\theta_{p}, \phi_{p}\right) \\
\vdots \\
\boldsymbol{w}_{o N}^{\mathrm{H}} \boldsymbol{a}_{N}\left(\theta_{p}, \phi_{p}\right)
\end{array}\right]\left[\begin{array}{c}
\boldsymbol{w}_{o 1}^{\mathrm{H}} \boldsymbol{a}_{1}\left(\theta_{p}, \phi_{p}\right) \\
\boldsymbol{w}_{o 2}^{\mathrm{H}} \boldsymbol{a}_{2}\left(\theta_{p}, \phi_{p}\right) \\
\vdots \\
\boldsymbol{w}_{o N}^{\mathrm{H}} \boldsymbol{a}_{N}\left(\theta_{p}, \phi_{p}\right)
\end{array}\right]^{\mathrm{H}},
$$

and

$$
\boldsymbol{R}_{n 2}=E\left\{\boldsymbol{y}_{n}(t) \boldsymbol{y}_{n}(t)^{\mathrm{H}}\right\}=\left[\begin{array}{ccc}
\boldsymbol{w}_{o 1}^{\mathrm{H}} E\left\{\boldsymbol{n}_{1}(t) \boldsymbol{n}_{1}(t)^{\mathrm{H}}\right\} \boldsymbol{w}_{o 1} & \ldots & \boldsymbol{w}_{o 1}^{\mathrm{H}} E\left\{\boldsymbol{n}_{1}(t) \boldsymbol{n}_{N}(t)^{\mathrm{H}}\right\} \boldsymbol{w}_{o N} \\
\vdots & \ddots & \vdots \\
\boldsymbol{w}_{o N}^{\mathrm{H}} E\left\{\boldsymbol{n}_{N}(t) \boldsymbol{n}_{N}(t)^{\mathrm{H}}\right\} \boldsymbol{w}_{o 1} & \cdots & \boldsymbol{w}_{o N}^{\mathrm{H}} E\left\{\boldsymbol{n}_{N}(t) \boldsymbol{n}_{N}(t)^{\mathrm{H}}\right\} \boldsymbol{w}_{o N}
\end{array}\right],
$$

respectively. According to the subarray beamforeming with successful interference suppression, the interference at the output of each subarray is negligible if the subarray size $L$ is appropriately chosen, e.g., $L>K$ for the considered case. Hence, we have the gain $\left|\boldsymbol{w}_{o l}^{\mathrm{H}} \boldsymbol{a}_{l}\left(\theta_{j}, \phi_{j}\right)\right| \approx 0$, for $l=1,2, \ldots, N, j=P+1, P+2, \ldots, K$, and an approximation as $\boldsymbol{y}(t) \approx \boldsymbol{y}_{S}(t)+\boldsymbol{y}_{n}(t)$. To further enhance the signal-to-noise ratio (SNR) at the UCA output, we formulate an optimization problem as

minimize $\boldsymbol{w}_{s}^{\mathrm{H}} \boldsymbol{R}_{n 2} \boldsymbol{w}_{s}, \quad$ subject to $\boldsymbol{G}_{s}^{\mathrm{H}} \boldsymbol{w}_{s}=\boldsymbol{c}$,

where $\boldsymbol{R}_{n 2}$ is given by (15), $\boldsymbol{G}_{s}=\left[\boldsymbol{z}\left(\theta_{1}, \phi_{1}\right) \boldsymbol{z}\left(\theta_{2}, \phi_{2}\right) \ldots \boldsymbol{z}\left(\theta_{P}, \phi_{P}\right)\right]$ is the constraint matrix for the virtual beamforming, the entries of $\boldsymbol{G}_{s}$ are given by $\boldsymbol{z}\left(\theta_{p}, \phi_{p}\right)=\left[\boldsymbol{w}_{o 1}^{\mathrm{H}} \boldsymbol{a}_{1}\left(\theta_{p}, \phi_{p}\right) \boldsymbol{w}_{o 2}^{\mathrm{H}} \boldsymbol{a}_{2}\left(\theta_{p}, \phi_{p}\right) \ldots \boldsymbol{w}_{o N}^{\mathrm{H}} \boldsymbol{a}_{N}\left(\theta_{p}, \phi_{p}\right)\right]^{\mathrm{T}}$ for $p=1,2, \ldots, P . w_{s}$ represents the virtual weight vector. The optimal solution for (16) is given by

$$
\boldsymbol{w}_{s o}=\boldsymbol{R}_{n 2}^{-1} \boldsymbol{G}_{s}\left(\boldsymbol{G}_{s}^{\mathrm{H}} \boldsymbol{R}_{n 2}^{-1} \boldsymbol{G}_{s}\right)^{-1} \boldsymbol{c} .
$$

Note that we have to find $\boldsymbol{R}_{n 2}$ since the noise at the virtual beamforming is no longer spatially white. Next, an efficient scheme is presented to estimate $\boldsymbol{R}_{n 2}$.

\subsection{Estimation of noise covariance matrix for the virtual beamforming}

Let $v_{n}$ be the noise component in the output $y_{n}(t)$ of the $n$th subarray. In matrix form, the received noise vector is expressed as

$$
\left[\begin{array}{llll}
v_{1} & v_{2} & \ldots & v_{N}
\end{array}\right]^{\mathrm{T}}=\left[\begin{array}{llll}
\boldsymbol{w}_{o 1}^{\mathrm{H}} \boldsymbol{n}_{1}(t) & \boldsymbol{w}_{o 2}^{\mathrm{H}} \boldsymbol{n}_{2}(t) \ldots \boldsymbol{w}_{o N}^{\mathrm{H}} \boldsymbol{n}_{N}(t)
\end{array}\right]^{\mathrm{T}} .
$$

The corresponding noise covariance matrix $\boldsymbol{R}_{n 2}$ is given by (15). For simplicity, we consider the case where the number of overlapped array sensors is $L-1$, i.e., $u=1$, as shown by Fig. 2 . Then, it is easy to show that the $i j$ th entry $R_{i j}=\boldsymbol{w}_{o i}^{\mathrm{H}} E\left\{\boldsymbol{n}_{i}(t) \boldsymbol{n}_{j}(t)^{\mathrm{H}}\right\} \boldsymbol{w}_{o j}$ of $\boldsymbol{R}_{n 2}$, for $i, j=1,2, \ldots, N$, is given as follows:

$$
\text { (i) } R_{i j}=\sigma_{n}^{2} \sum_{l=1}^{L} w_{o i l}^{*} w_{o j l}
$$

since $E\left\{\boldsymbol{n}_{i}(t) \boldsymbol{n}_{j}(t)^{\mathrm{H}}\right\}=\sigma_{n}^{2} \boldsymbol{I}_{L}$ for $i=j$, where the superscript “*” denotes complex conjugate. $w_{o i l}$ and $w_{o j l}$ are the $l$ th entries of $\boldsymbol{w}_{o i}$ and $\boldsymbol{w}_{o j}$, respectively.

(ii) $\quad R_{i j}=\sigma_{n}^{2} \sum_{l=1}^{L-1} w_{o i(l+1)}^{*} w_{o j l} \quad$ for $i-j=1$.

(iii) $\quad R_{i j}=\sigma_{n}^{2}\left(\sum_{l=1}^{L-1} w_{o j(l+1)}^{*} w_{o i l}\right)^{*}$ for $i-j=-1$.

(iv) $R_{i j}=\sigma_{n}^{2} \sum_{l=1}^{L-d} w_{o i(l+d)}^{*} w_{o j l} \quad$ if $d<L \quad$ and $\quad R_{i j}=0 \quad$ otherwise for $i-j=d>0$.

(v) $\quad R_{i j}=\sigma_{n}^{2}\left(\sum_{l=1}^{L-d} w_{o j(l+d)}^{*} w_{o i l}\right)^{*} \quad$ if $d<L \quad$ and $\quad R_{i j}=0 \quad$ otherwise $\quad$ for $i-j=-d \leqslant 0$. 
The value of $w_{o i l}$ which is the $l$ th entry of $\boldsymbol{w}_{o i}, l=1,2, \ldots, L$, can be obtained from (11). As to $\sigma_{n}^{2}$, we present an appropriate scheme to estimate $\sigma_{n}^{2}$ if it is not available in practice. First, we estimate the number $K$ of signal sources from the received array data vector. Among the techniques available for this purpose, we adopt the most notable one, namely, the AIC or MDL criteria proposed by [21]. Then, we perform the eigenvalue decomposition (EVD) of the correlation matrix $\boldsymbol{R}_{1}$ of the first subarray data vector $\boldsymbol{x}_{1}(t)$ shown by (9) and find the corresponding eigenvalues $\lambda_{i}$ in descending order for $i=1,2, \ldots, L$. Finally, we take the average of the smallest $L-\hat{K}$ eigenvalues as the estimate $\hat{\sigma}_{n}^{2}$ of $\sigma_{n}^{2}$, where $\hat{K}$ is the estimate of $K$. According to the theoretical results regarding the AIC or MDL algorithm presented by Wax et al. [21], it has been shown that the MDL criterion yields a consistent estimate of the number of signals, while the AIC yields an inconsistent estimate that tends, asymptotically, to overestimate the number of signals. Our simulation experience shows that the MDL criterion provides the correct estimate and the AIC criterion provides either the correct estimate or over-estimate under the situations like Examples 1 and 2 of the paper. The performance of the proposed beamformer under that the SNR of the signal sources is as low as $0 \mathrm{~dB}$ with several hundred data snapshots is not sensitive to the effect of $K$, when it is over-estimate. The output $f(t)$ of the UCA is given by

$$
f(t)=\boldsymbol{w}_{s o}^{\mathrm{H}}\left[\boldsymbol{w}_{o 1}^{\mathrm{H}} \boldsymbol{x}_{1}(t) \boldsymbol{w}_{o 2}^{\mathrm{H}} \boldsymbol{x}_{2}(t) \ldots \boldsymbol{w}_{o N}^{\mathrm{H}} \boldsymbol{x}_{N}(t)\right]^{\mathrm{T}}=\sum_{n=1}^{N} w_{n}^{*} \boldsymbol{w}_{o n}^{\mathrm{H}} \boldsymbol{x}_{n}(t),
$$

where $w_{l}$ denotes the $l$ th entry of $\boldsymbol{w}_{s o}$ for $l=1,2, \ldots, N$. In matrix form, (24) can be expressed as

$$
f(t)=\left[w_{1}\left[\begin{array}{c}
w_{o 11} \\
w_{o 12} \\
\vdots \\
w_{o 1 L} \\
0 \\
\vdots \\
0 \\
0
\end{array}\right]+w_{2}\left[\begin{array}{c}
0 \\
w_{o 21} \\
w_{o 22} \\
\vdots \\
w_{o 2 L} \\
0 \\
\vdots \\
0
\end{array}\right]+\cdots+w_{N}\left[\begin{array}{c}
w_{o N 2} \\
w_{o N 3} \\
\vdots \\
w_{o N L} \\
0 \\
\vdots \\
0 \\
w_{o N 1}
\end{array}\right]\right]^{\mathrm{H}} \boldsymbol{x}(t) .
$$

From (25), the equivalent overall $M \times 1$ optimal weight vector is given by

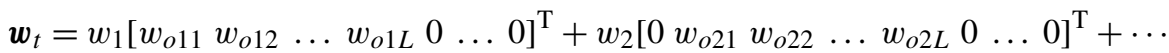

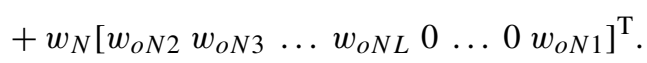

The power gain $G(\theta, \phi)$ in the direction vector $\boldsymbol{a}(\theta, \phi)$ is given by

$$
G(\theta, \phi)=10 \log _{10}\left|\boldsymbol{w}_{t}^{\mathrm{H}} \boldsymbol{a}(\theta, \phi)\right|^{2}=10 \log _{10}\left|\sum_{n=1}^{N} w_{n}^{*} \boldsymbol{w}_{o n}^{\mathrm{H}} \boldsymbol{a}_{n}(\theta, \phi)\right|^{2} .
$$

In the presence of coherent interference, the effectiveness of the UCA beamformer will be deteriorated because the coherence between the desired signals and interferers will reduce the rank of $\boldsymbol{A}_{l} \boldsymbol{R}_{s} A_{l}^{\mathrm{H}}$ given by (10) and, hence, destroy the eigenstructure of $\boldsymbol{R}_{l}$, for $l=1,2, \ldots, N$. To restore the desired eigenstructure of $\boldsymbol{R}_{l}$, we compute the optimal weight vector by replacing each $\boldsymbol{R}_{l}$ of (11) with the averaged one $\hat{\boldsymbol{R}}$

$$
\hat{\boldsymbol{R}}=\frac{1}{N} \sum_{l=1}^{N} \boldsymbol{R}_{l} .
$$

The matrix $\hat{\boldsymbol{R}}$ is also used instead of each $\boldsymbol{R}_{l}$ for finding the estimates $\hat{K}$ and $\hat{\sigma}_{n}^{2}$.

\section{Theoretical analysis}

Here, we investigate the matrix $\boldsymbol{A}=\left[\boldsymbol{a}\left(\theta_{1}, \phi_{1}\right) \boldsymbol{a}\left(\theta_{2}, \phi_{2}\right) \ldots \boldsymbol{a}\left(\theta_{K}, \phi_{K}\right)\right]$ with the direction vector $\boldsymbol{a}\left(\theta_{k}, \phi_{k}\right)$ of the $k$ th signal given by (1). The following theorem shows that the direction vectors with the same elevation angle and distinct azimuth angles are linearly independent. 
Theorem 1. Let the $K$ narrow-band and far-field signals be impinging on the array with angles $\left(\theta_{k}, \phi_{k}\right), k=$ $1,2, \ldots, K$, have the same elevation angle $\theta_{k}=\theta$ and distinct azimuth angles $\phi_{k}$, for $k=1,2, \ldots, K$. Then, the matrix $\boldsymbol{A}_{s}=\left[\boldsymbol{a}\left(\theta, \phi_{1}\right) \boldsymbol{a}\left(\theta, \phi_{2}\right) \ldots \boldsymbol{a}\left(\theta, \phi_{K}\right)\right]$ with the direction vector $\boldsymbol{a}\left(\theta, \phi_{k}\right)$ of the $k$ th signal given by (1) has rank equal to $K$.

Proof. Consider the phase delay at the $m$ th array sensor, which is given by (2)

$$
a_{m}\left(\theta_{k}, \phi_{k}\right)=\exp \left\{j 2 \pi \frac{R}{\lambda} \cos \left[\phi_{k}-\frac{2 \pi(m-1)}{M}\right] \sin \left(\theta_{k}\right)\right\} .
$$

Let the elevation angle $\theta_{k}=\theta$ and $2 \pi \sin (\theta) / \lambda=q$. The phase delay becomes

$$
a_{m}\left(\theta, \phi_{k}\right)=\exp \left\{j q R \cos \left[\phi_{k}-\frac{2 \pi(m-1)}{M}\right]\right\} .
$$

Hence, the matrix $\boldsymbol{A}_{s}=\left[\boldsymbol{a}\left(\theta, \phi_{1}\right) \boldsymbol{a}\left(\theta, \phi_{2}\right) \ldots \boldsymbol{a}\left(\theta, \phi_{K}\right)\right]$ can be expressed by

$$
\boldsymbol{B}=\left[\begin{array}{cccc}
a_{1}\left(\phi_{1}\right) & a_{1}\left(\phi_{2}\right) & \ldots & a_{1}\left(\phi_{K}\right) \\
a_{2}\left(\phi_{1}\right) & a_{2}\left(\phi_{2}\right) & \ldots & a_{2}\left(\phi_{K}\right) \\
\vdots & \vdots & \ddots & \vdots \\
a_{M}\left(\phi_{1}\right) & a_{M}\left(\phi_{2}\right) & \ldots & a_{M}\left(\phi_{K}\right)
\end{array}\right]=\left[\boldsymbol{b}\left(\phi_{1}\right) \boldsymbol{b}\left(\phi_{2}\right) \ldots \boldsymbol{b}\left(\phi_{K}\right)\right]
$$

Using the modal transformation [1], we create a $(2 h+1) \times M$ transformation matrix $\boldsymbol{T}$ as follows:

$$
\boldsymbol{T}=\frac{1}{\sqrt{M}}\left[\begin{array}{ccccc}
1 & T^{-h} & T^{-2 h} & \ldots & T^{-(M-1) h} \\
\vdots & \vdots & \vdots & \ldots & \vdots \\
1 & T^{-1} & T^{-2} & \ldots & T^{-(M-1)} \\
1 & 1 & 1 & \ldots & 1 \\
1 & T^{1} & T^{2} & \ldots & T^{(M-1)} \\
\vdots & \vdots & \vdots & \ldots & \vdots \\
1 & T^{h} & T^{2 h} & \ldots & T^{(M-1) h}
\end{array}\right]
$$

where $(2 h+1) \leqslant M$ and $T$ is given by $T=\exp (j 2 \pi / M)$. After transformation, the entry $\boldsymbol{b}\left(\phi_{k}\right)$ of $\boldsymbol{B}$ shown by (30) becomes

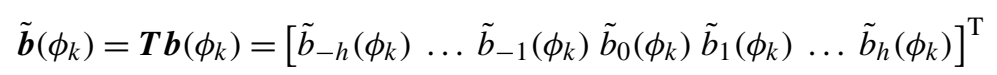

for $k=1,2, \ldots, K$, where $\tilde{b}_{\ell}\left(\phi_{k}\right)$ is given by

$$
\tilde{b}_{\ell}\left(\phi_{k}\right)=\frac{1}{\sqrt{M}} \sum_{m=0}^{M-1}\left\{\exp (j 2 \pi m \ell / M) \times \exp \left[j q R \cos \left(\phi_{k}-2 \pi m / M\right)\right]\right\}
$$

for $\ell=-h, \ldots,-1,0,1, \ldots, h$. Since the term $\exp \left[j q R \cos \left(\phi_{k}-2 \pi m / M\right)\right]$ is a periodic function, it can be expressed by a Fourier series expansion as follows:

$$
\exp \left[j q R \cos \left(\phi_{k}-2 \pi m / M\right)\right]=\sum_{n=-\infty}^{\infty} j^{n} J_{n}(q R) \exp \left[j n\left(\phi_{k}-2 \pi m / M\right)\right],
$$

where $J_{n}$ represents the first-kind Bessel function with order $n$. Substituting (34) into (33) yields

$$
\tilde{b}_{\ell}\left(\phi_{k}\right)=\frac{1}{\sqrt{M}} \sum_{n=-\infty}^{\infty} j^{n} J_{n}(q R) \exp \left(j n \phi_{k}\right) \sum_{m=0}^{M-1}\{\exp (j 2 \pi m \ell / M-j 2 \pi m n / M)\} .
$$


Applying the result

$$
\sum_{m=0}^{M-1}\{\exp (j 2 \pi m \ell / M-j 2 \pi m n / M)\}= \begin{cases}M, & \text { if } n-\ell=i M, i=\text { integer, } \\ 0, & \text { otherwise, }\end{cases}
$$

we rewrite (35) as

$$
\tilde{b}_{\ell}\left(\phi_{k}\right)=\sqrt{M} \sum_{i=-\infty}^{\infty} j^{\ell+i M} J_{\ell+i M}(q R) \exp \left[j(\ell+i M) \phi_{k}\right] .
$$

In the case of $M \gg q R$, the dominant term in the infinite series of (37) is the one corresponding to $i=0$ while all the other terms corresponding to $i \neq 0$ can be neglected. Moreover, the asymptotic property of the Bessel function $J_{n}(q R)$ for $n \gg q R$ provides the following approximation

$$
J_{n}(q R) \approx \frac{1}{\sqrt{2 \pi n}}\left(\frac{e q R}{2 n}\right)^{n}
$$

An appropriate approximation expression for (37) is given by

$$
\tilde{b}_{\ell}\left(\phi_{k}\right) \approx \sqrt{M} j^{\ell} J_{\ell}(q R) \exp \left(j \ell \phi_{k}\right)
$$

for $\ell=-h, \ldots,-1,0,1, \ldots, h$, if $M \gg q R$. (39) reveals that $\tilde{b}_{\ell}\left(\phi_{k}\right)$ possesses a phase $\ell \phi_{k}$ depending on the signal angle $\phi_{k}$ and a factor $\sqrt{M} j^{\ell} J_{\ell}(q R)$ independent of $\phi_{k}$. A $(2 h+1) \times(2 h+1)$ diagonal matrix $\boldsymbol{U}$ is formed to preserve only the source angle-dependent phase for (39):

$$
\boldsymbol{U}=\sqrt{M}\left[\begin{array}{ccccc}
j^{-h} J_{-h}(q R) & 0 & \cdots & 0 & 0 \\
0 & \ddots & 0 & \cdots & 0 \\
\vdots & 0 & j^{0} J_{0}(q R) & 0 & \vdots \\
\vdots & \vdots & 0 & \ddots & 0 \\
0 & \cdots & \cdots & 0 & j^{h} J_{h}(q R)
\end{array}\right] .
$$

From (30), (31), (39), and (40), we construct a $(2 h+1) \times K$ matrix $\overline{\boldsymbol{B}}$ containing the transformed direction vectors with only the source angle-dependent phase as follows:

$$
\boldsymbol{T} \boldsymbol{B} \approx \boldsymbol{U} \overline{\boldsymbol{B}},
$$

where $\overline{\boldsymbol{B}}$ has the form given by

$$
\overline{\boldsymbol{B}}=\left[\begin{array}{cccc}
\exp \left(-h \phi_{1}\right) & \exp \left(-h \phi_{2}\right) & \cdots & \exp \left(-h \phi_{K}\right) \\
\vdots & \vdots & \cdots & \vdots \\
\exp \left(-\phi_{1}\right) & \exp \left(-\phi_{2}\right) & \cdots & \exp \left(-\phi_{2}\right) \\
1 & 1 & \cdots & 1 \\
\exp \left(\phi_{1}\right) & \exp \left(\phi_{2}\right) & \cdots & \exp \left(\phi_{2}\right) \\
\vdots & \vdots & \cdots & \vdots \\
\exp \left(h \phi_{1}\right) & \exp \left(h \phi_{2}\right) & \cdots & \exp \left(h \phi_{K}\right)
\end{array}\right]
$$

In order to ensure that the transformation given by (41) is useful, the conditions of $J_{\ell}(q R) \neq 0$, for $\ell=-h, \ldots,-1$, $0,1, \ldots, h$, must be guaranteed. Accordingly, we note from (40) and (42) that $\boldsymbol{U}$ and $\overline{\boldsymbol{B}}$ have ranks equal to $(2 h+1)$ and $K$, respectively. In contrast, the matrix $\boldsymbol{T}$ of (31) has rank equal to $(2 h+1)$. Therefore, it is clear from (41) that the rank of $\boldsymbol{B}$ must be $K$ which is the number of signal sources. Hence, the matrix $\boldsymbol{A}_{s}=\left[\boldsymbol{a}\left(\theta, \phi_{1}\right) \boldsymbol{a}\left(\theta, \phi_{2}\right) \ldots \boldsymbol{a}\left(\theta, \phi_{K}\right)\right]$ with the direction vector $\boldsymbol{a}\left(\theta, \phi_{k}\right)$ of the $k$ th signal has rank equal to $K$. This completes the proof.

Theorem 2. The averaged correlation matrix $\frac{1}{N} \sum_{l=1}^{N} \boldsymbol{A}_{l} \boldsymbol{R}_{s} \boldsymbol{A}_{l}^{\mathrm{H}}$ of $(28)$ has rank equal to min $(L, N)$.

Proof. From (9), we note that each subarray has size $L$ and the number of array sensors overlapped for two adjacent subarrays is $L-u$. Hence, $\hat{\boldsymbol{R}}$ can be rewritten as follows: 


$$
\hat{\boldsymbol{R}}=\frac{1}{N} \sum_{l=1}^{N} \boldsymbol{R}_{l}=\frac{1}{N} \sum_{l=1}^{N} \boldsymbol{A}_{l} \boldsymbol{R}_{s} \boldsymbol{A}_{l}^{\mathrm{H}}+\sigma_{n}^{2} \boldsymbol{I}_{L},
$$

where $\boldsymbol{R}_{s}=E\left\{\boldsymbol{s}(t) \boldsymbol{s}(t)^{\mathrm{H}}\right\}$ denotes the signal correlation matrix and $\boldsymbol{A}_{l}=\left[\boldsymbol{a}_{l}\left(\theta_{1}, \phi_{1}\right) \boldsymbol{a}_{l}\left(\theta_{2}, \phi_{2}\right) \ldots \boldsymbol{a}_{l}\left(\theta_{K}, \phi_{K}\right)\right]$ the $L \times K$ matrix with the direction vector of the $k$ th signal given by $\boldsymbol{a}_{l}\left(\theta_{k}, \phi_{k}\right)=\left[a_{(l-1) n+1}\left(\theta_{k}, \phi_{k}\right) a_{(l-1) n+2}\left(\theta_{k}, \phi_{k}\right) \ldots\right.$ $\left.a_{(l-1) n+L}\left(\theta_{k}, \phi_{k}\right)\right]^{\mathrm{T}}$. Hence, $\boldsymbol{A}_{l}$ can be rewritten by

$$
\boldsymbol{A}_{l}=\left[\begin{array}{cccc}
a_{(l-1) n+1}\left(\theta_{1}, \phi_{1}\right) & a_{(l-1) n+1}\left(\theta_{2}, \phi_{2}\right) & \ldots & a_{(l-1) n+1}\left(\theta_{K}, \phi_{K}\right) \\
a_{(l-1) n+2}\left(\theta_{1}, \phi_{1}\right) & a_{(l-1) n+2}\left(\theta_{2}, \phi_{2}\right) & \ldots & a_{(l-1) n+2}\left(\theta_{K}, \phi_{K}\right) \\
\vdots & \vdots & \ddots & \vdots \\
a_{(l-1) n+L}\left(\theta_{1}, \phi_{1}\right) & a_{(l-1) n+L}\left(\theta_{2}, \phi_{2}\right) & \ldots & a_{(l-1) n+L}\left(\theta_{K}, \phi_{K}\right)
\end{array}\right] .
$$

Accordingly, the first term in (43) can be expressed as follows:

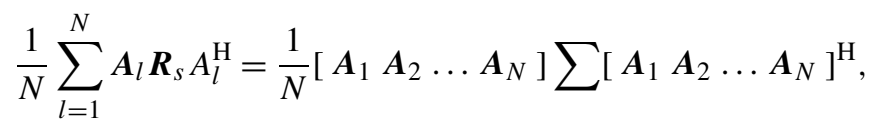

where $\Sigma$ is a block-diagonal $N K \times N K$ matrix given by

$$
\Sigma=\left[\begin{array}{cccc}
\boldsymbol{R}_{s} & \mathbf{0} & \ldots & \mathbf{0} \\
\mathbf{0} & \boldsymbol{R}_{s} & \ldots & \mathbf{0} \\
\vdots & \vdots & \ddots & \vdots \\
\mathbf{0} & \ldots & \mathbf{0} & \boldsymbol{R}_{s}
\end{array}\right]
$$

Next, we consider the worst case for the coherent problem. Let all of the signal sources be coherent. Then, the matrix $\boldsymbol{R}_{S}$ has rank equal to one and can be decomposed as [22]

$$
\boldsymbol{R}_{s}=\left(\boldsymbol{R}_{s}\right)^{1 / 2}\left(\boldsymbol{R}_{s}^{\mathrm{H}}\right)^{1 / 2}=\boldsymbol{r} \boldsymbol{r}^{\mathrm{H}},
$$

where the $K \times 1$ vector $\boldsymbol{r}=\left[\begin{array}{llll}r_{1} & r_{2} & \ldots & r_{K}\end{array}\right]^{\mathrm{T}}$ with $r_{k} \neq 0, k=1,2, \ldots, K$. Since the rank of $\boldsymbol{Z} \boldsymbol{Z}^{\mathrm{H}}$ (denoted as $\operatorname{rank}\left(\boldsymbol{Z} \boldsymbol{Z}^{\mathrm{H}}\right)$ ) for a nonzero matrix $\boldsymbol{Z}$ is equal to $\operatorname{rank}(\boldsymbol{Z})$ [22], we have

$$
\begin{aligned}
& \operatorname{rank}\left(\left[\begin{array}{llll}
\boldsymbol{A}_{1} & \boldsymbol{A}_{2} & \ldots & \boldsymbol{A}_{N}
\end{array}\right] \Sigma\left[\begin{array}{llll}
\boldsymbol{A}_{1} & \boldsymbol{A}_{2} & \ldots & \boldsymbol{A}_{N}
\end{array}\right]^{\mathrm{H}}\right)=\operatorname{rank}\left(\left[\begin{array}{cccc}
\boldsymbol{A}_{1} & \boldsymbol{A}_{2} & \ldots & \boldsymbol{A}_{N}
\end{array}\right]\left[\begin{array}{cccc}
\boldsymbol{r} & \mathbf{0} & \ldots & \mathbf{0} \\
\mathbf{0} & \boldsymbol{r} & \ldots & \vdots \\
\vdots & \ldots & \ddots & \mathbf{0} \\
\mathbf{0} & \ldots & \mathbf{0} & \boldsymbol{r}
\end{array}\right]\right) \\
& =\operatorname{rank}\left(\left[\begin{array}{llll}
\boldsymbol{A}_{1} \boldsymbol{r} & \boldsymbol{A}_{2} \boldsymbol{r} & \ldots & \boldsymbol{A}_{N} \boldsymbol{r}
\end{array}\right]\right) .
\end{aligned}
$$

Moreover, the $L \times N$ matrix $\left[\begin{array}{llll}\boldsymbol{A}_{1} \boldsymbol{r} & \boldsymbol{A}_{2} \boldsymbol{r} & \ldots & \boldsymbol{A}_{N} \boldsymbol{r}\end{array}\right]$ can be rewritten by

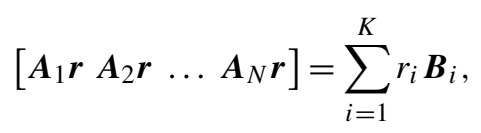

where the $L \times N$ matrix $\boldsymbol{B}_{i}$ is given by

$$
\begin{aligned}
& \boldsymbol{B}_{i}=\left[\begin{array}{cccc}
a_{1}\left(\theta_{i}, \phi_{i}\right) & a_{n+1}\left(\theta_{i}, \phi_{i}\right) & \ldots & a_{(N-1) n+1}\left(\theta_{i}, \phi_{i}\right) \\
a_{2}\left(\theta_{i}, \phi_{i}\right) & a_{n+2}\left(\theta_{i}, \phi_{i}\right) & \ldots & a_{(N-1) n+2}\left(\theta_{i}, \phi_{i}\right) \\
\vdots & \vdots & \ddots & \vdots \\
a_{L}\left(\theta_{i}, \phi_{i}\right) & a_{n+L}\left(\theta_{i}, \phi_{i}\right) & \ldots & a_{(N-1) n+L}\left(\theta_{i}, \phi_{i}\right)
\end{array}\right] \\
& =\left[\begin{array}{cccc}
a_{1}\left(\theta_{i}, \phi_{i}\right) & a_{n+1}\left(\theta_{i}, \phi_{i}\right) & \ldots & a_{(N-1) n+1}\left(\theta_{i}, \phi_{i}\right) \\
a_{1}\left(\theta_{i}, \phi_{i}-2 \pi / M\right) & a_{n+1}\left(\theta_{i}, \phi_{i}-2 \pi / M\right) & \ldots & a_{(N-1) n+1}\left(\theta_{i}, \phi_{i}-2 \pi / M\right) \\
\vdots & \vdots & \ddots & \vdots \\
a_{1}\left(\theta_{i}, \phi_{i}-2 \pi(L-1) / M\right) & a_{n+1}\left(\theta_{i}, \phi_{i}-2 \pi(L-1) / M\right) & \ldots & a_{(N-1) n+1}\left(\theta_{i}, \phi_{i}-2 \pi(L-1) / M\right)
\end{array}\right] .
\end{aligned}
$$




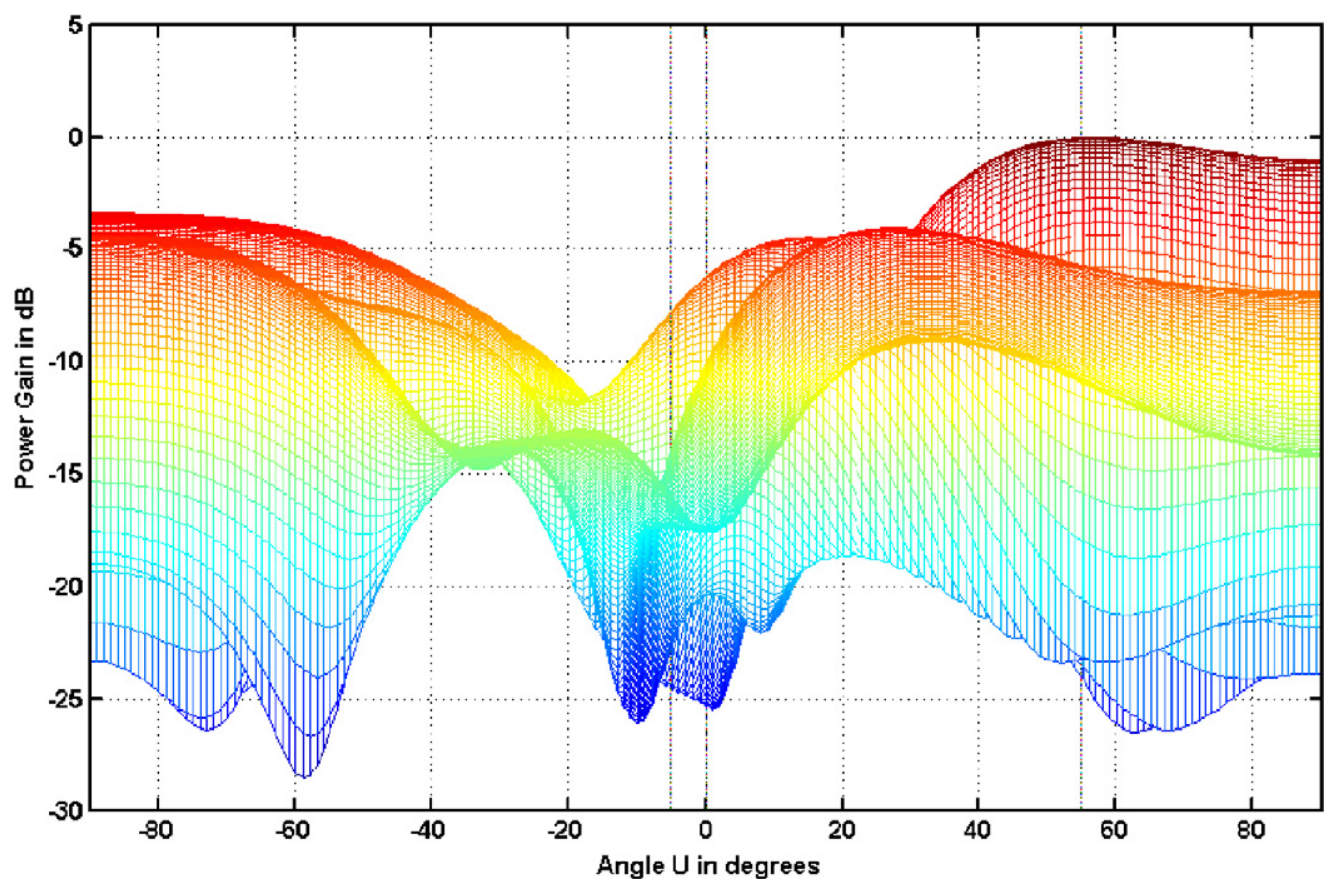

(a)

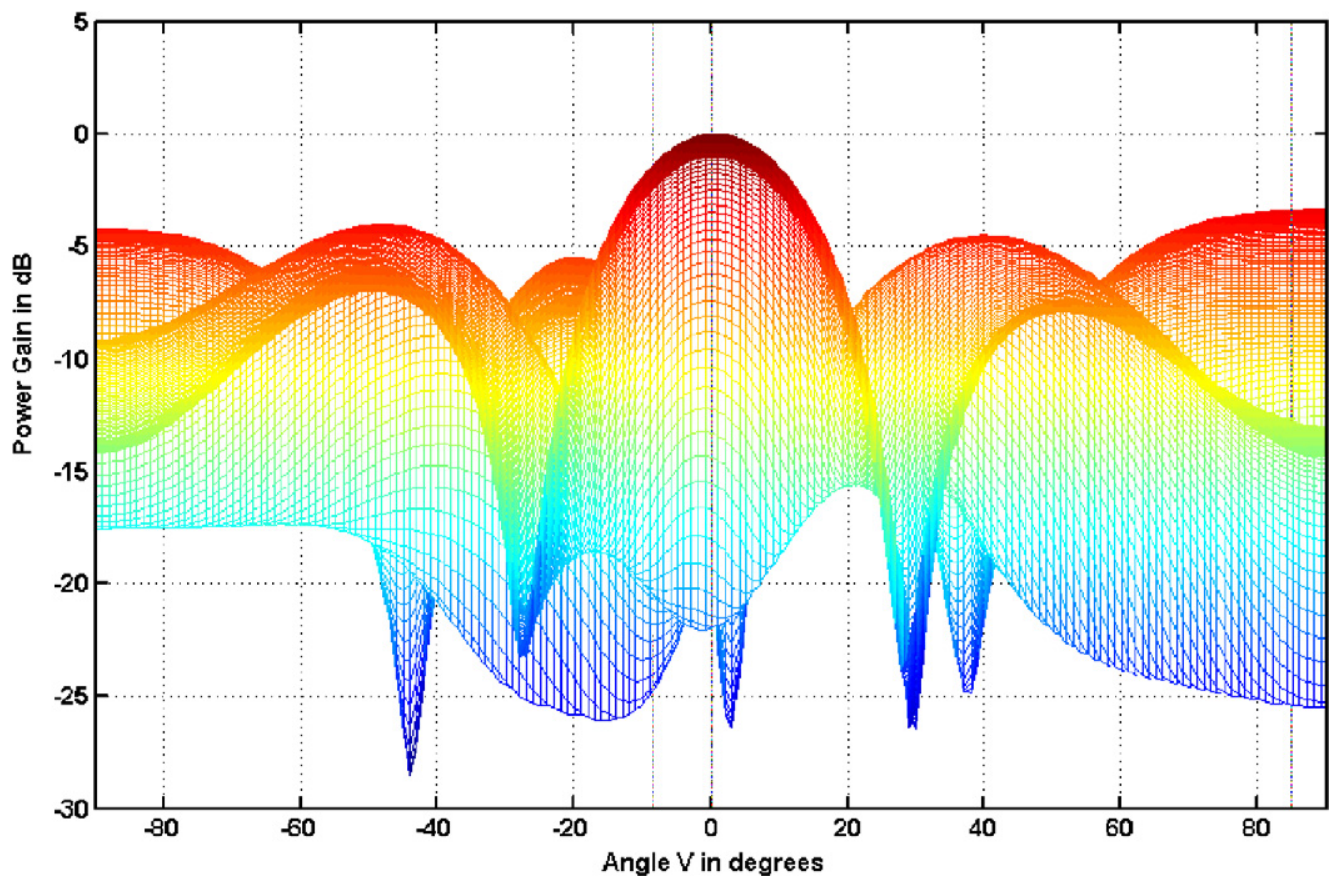

(b)

Fig. 3. Beam pattern without coherence using proposed method for Example 1.

Equivalently, we can view that the $N \times L$ matrix $\boldsymbol{B}_{i}^{\mathrm{T}}$ contains the direction vector $\tilde{\boldsymbol{a}}\left(\theta_{i}, \varphi_{\ell}\right)=\left[a_{1}\left(\theta_{i}, \varphi_{\ell}\right) a_{n+1}\left(\theta_{i}\right.\right.$, $\left.\left.\varphi_{\ell}\right) \ldots a_{(N-1) n+1}\left(\theta_{i}, \varphi_{\ell}\right)\right]^{\mathrm{T}}$ of the $\ell$ th signal with angle $\left(\theta_{i}, \varphi_{\ell}\right), \ell=1,2, \ldots, L$, where $\varphi_{\ell}=\phi_{i}-2 \pi(\ell-1) / M$, and all of the $L$ signals have the same elevation angle $\theta_{i}$. Applying Theorem 1, we note that each $\boldsymbol{B}_{i}$ possesses rank equal to $\min (L, N), i=1,2, \ldots, K$. As a result, the rank of (45) is equal to $\operatorname{rank}\left(\sum_{i=1}^{K} r_{i} \boldsymbol{B}_{i}\right)$ which is given by $\min (L, N)$. This completes the proof. 


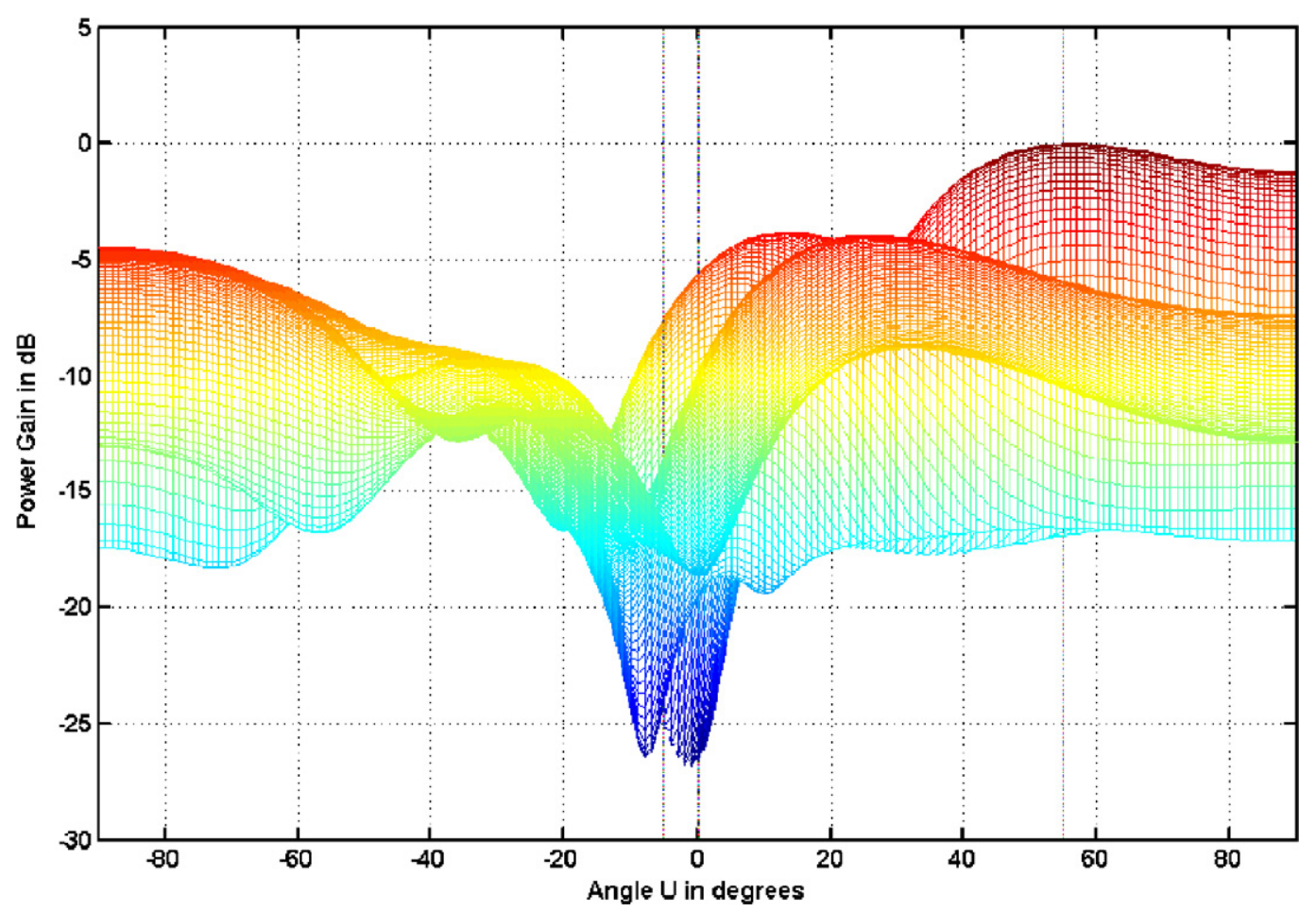

(a)

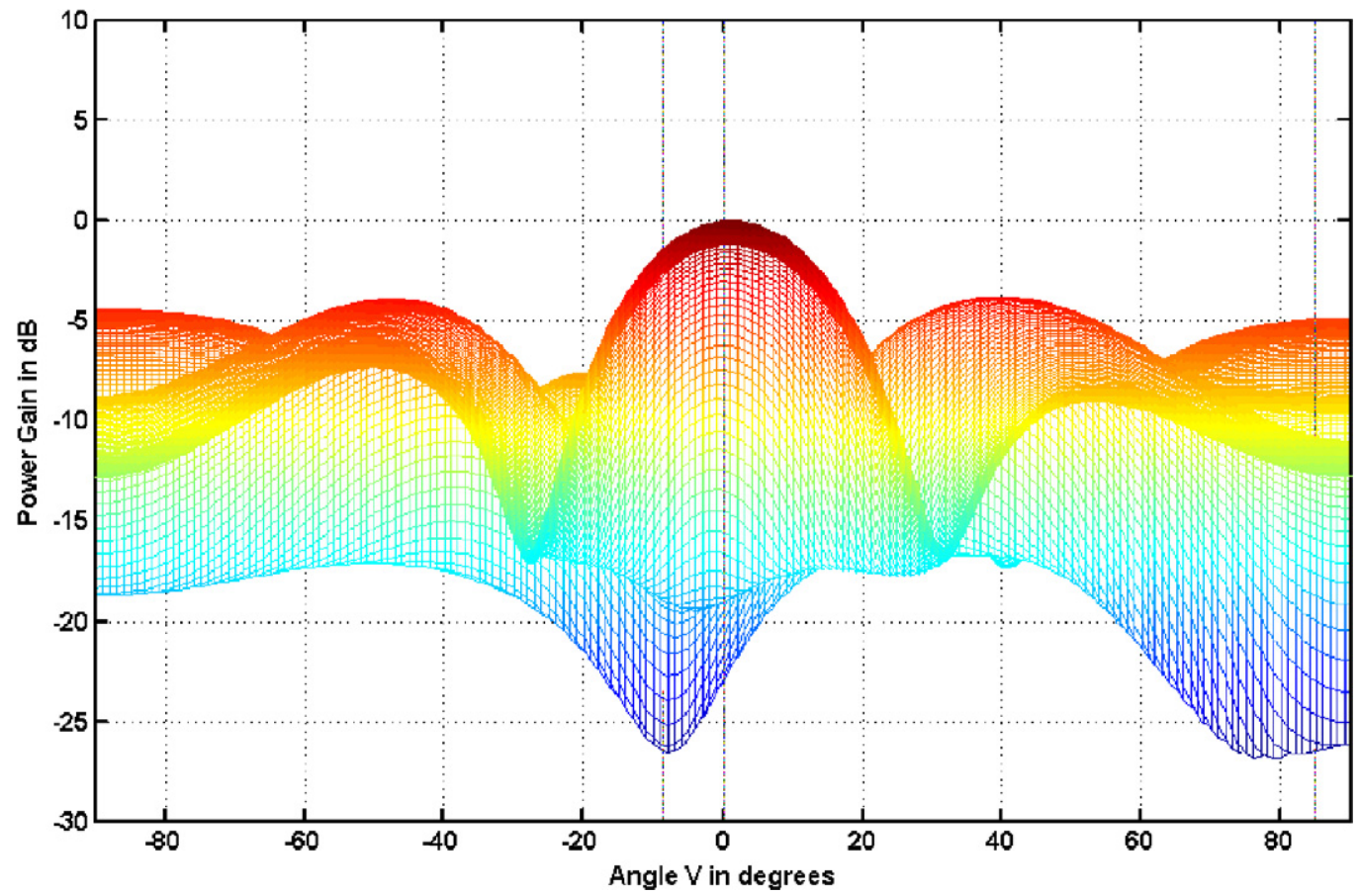

(b)

Fig. 4. Beam pattern without coherence using conventional method for Example 1.

Theorem 2 shows that $\operatorname{rank}(\hat{\boldsymbol{R}})$ can be greater than or equal to $K$ which is the number of signal sources if we set $\min (L, N)$ greater than or equal to $K$. In other words, the rank of the correlation matrix $\boldsymbol{R}_{x}$ can be restored by applying the proposed method. 


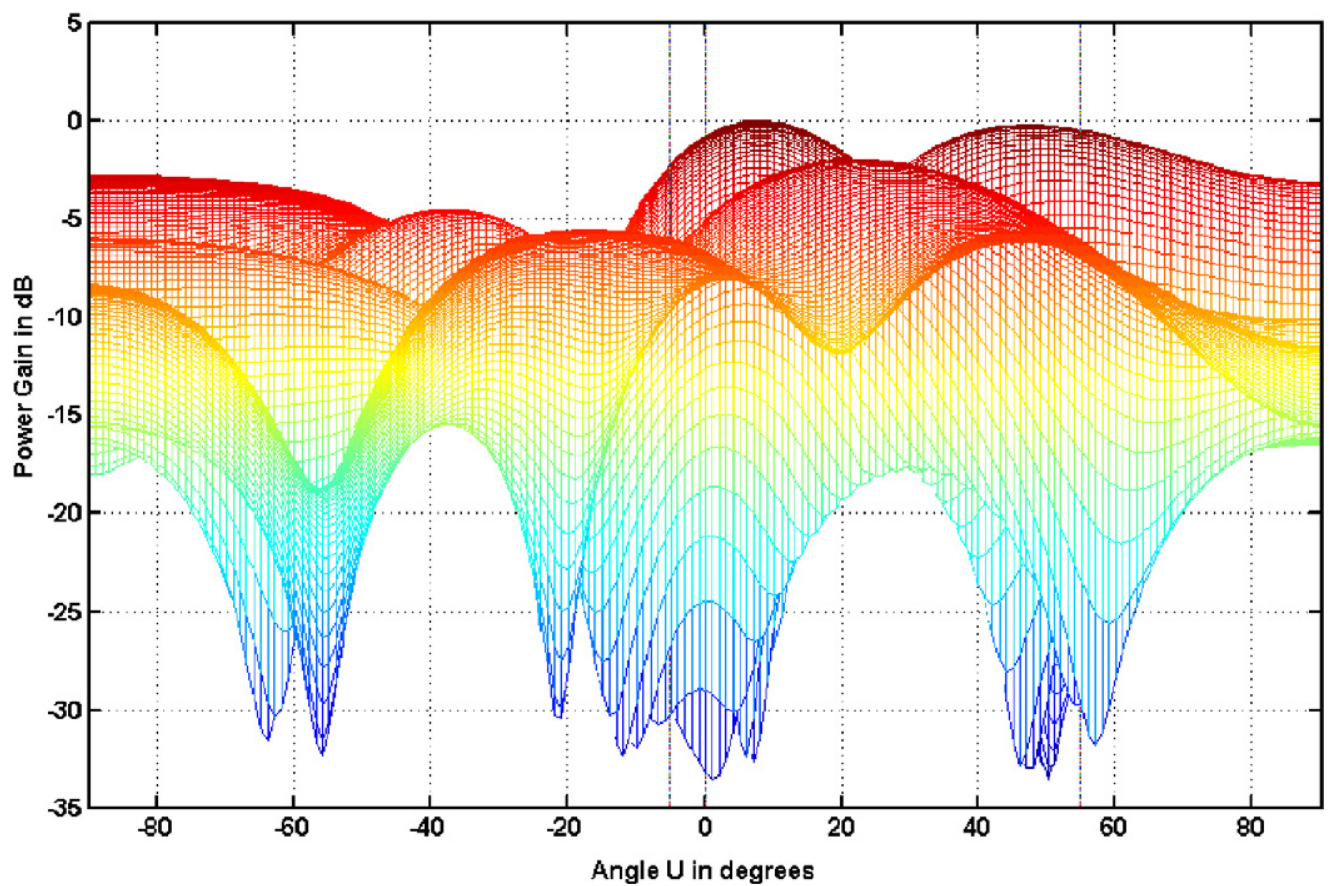

(a)

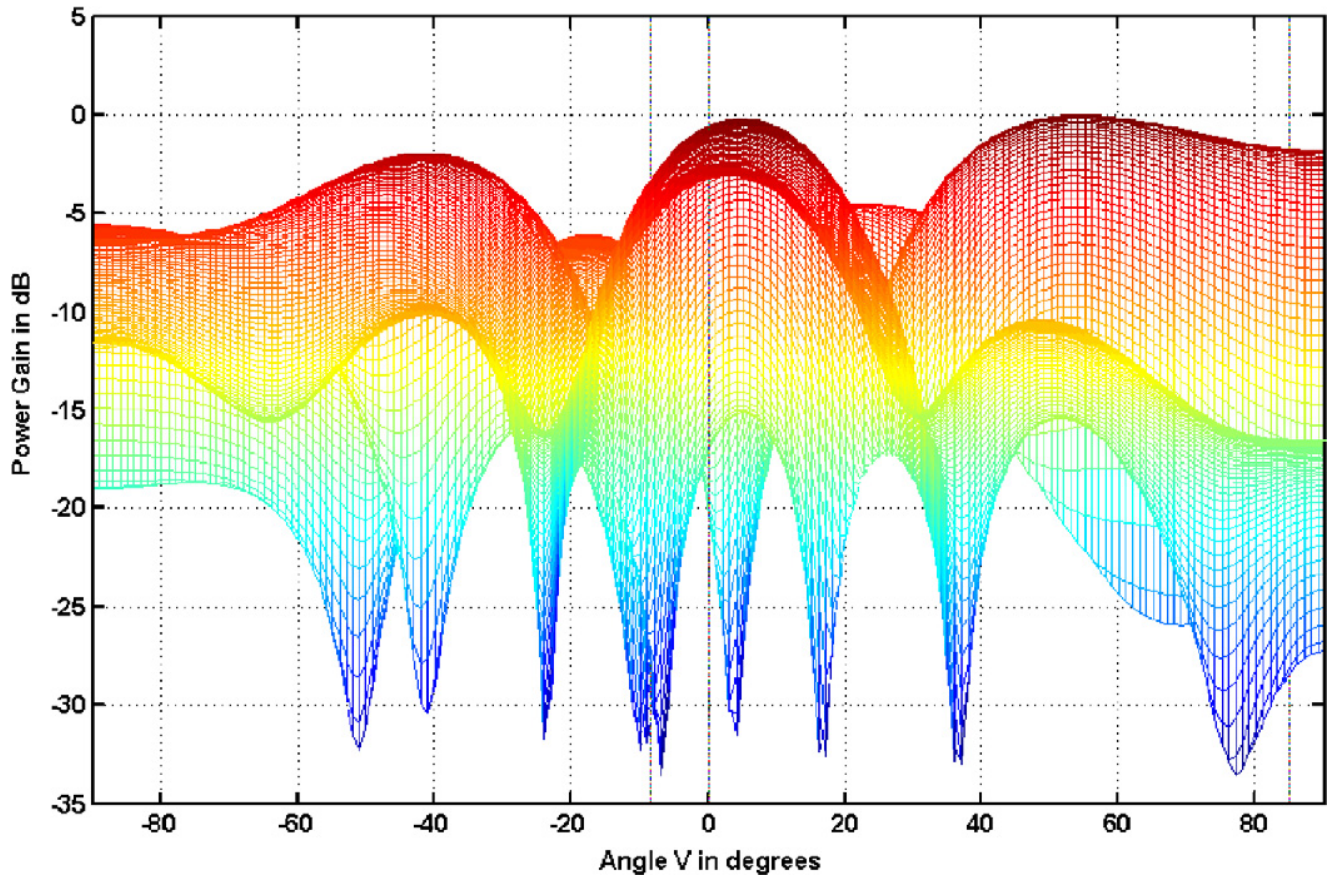

(b)

Fig. 5. Beam pattern with coherence using proposed method for Example 1.

\section{Simulation examples}

For simulations, we use a UCA with the ratio of inter-element spacing $d$ to the wavelength $\lambda$ of the desired signals equal to 0.5 . The simulation results are obtained by using 500 data snapshots and averaging 100 independent runs with independent noise samples for each run. All signals used are binary phase shift keying (BPSK) signals with 


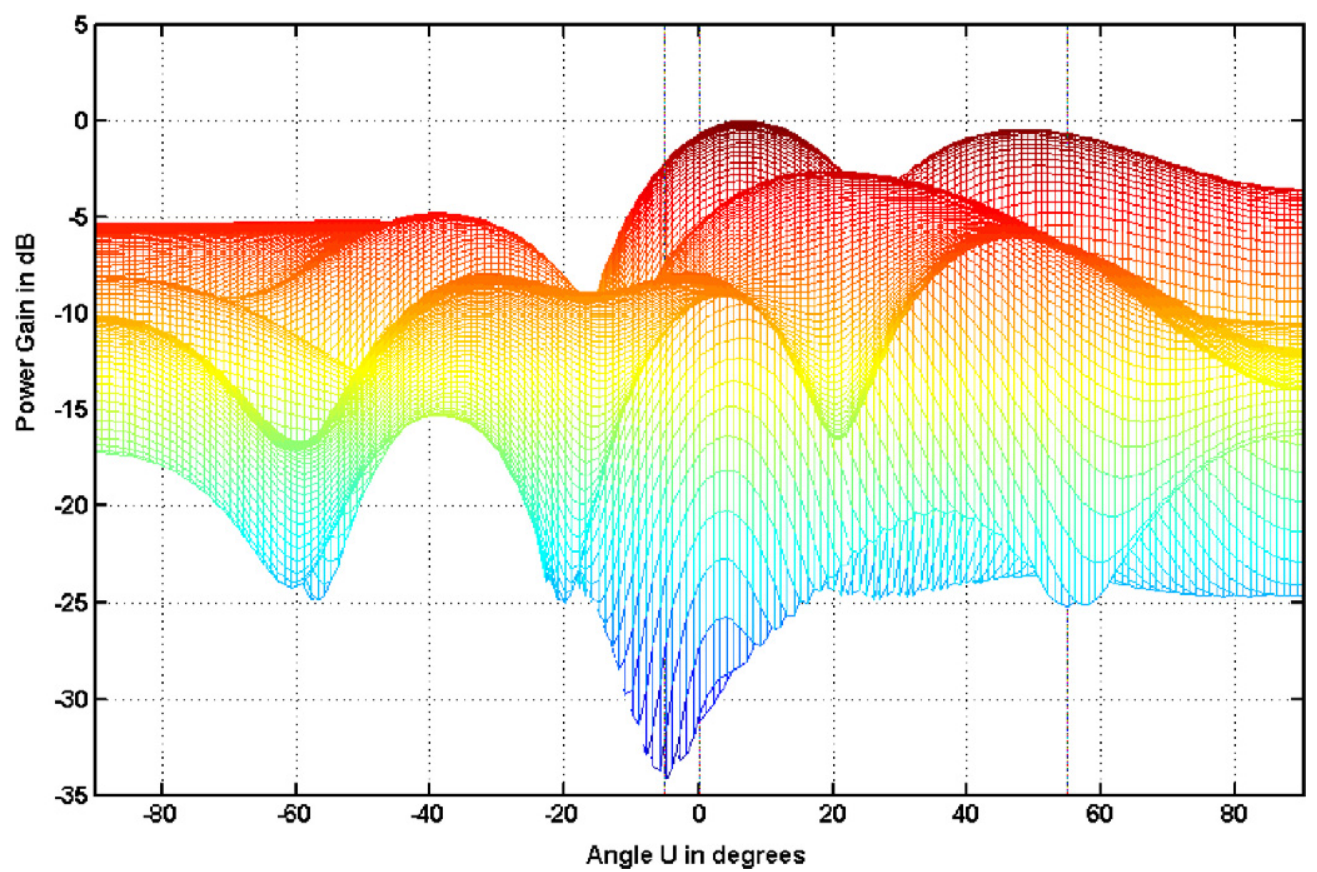

(a)

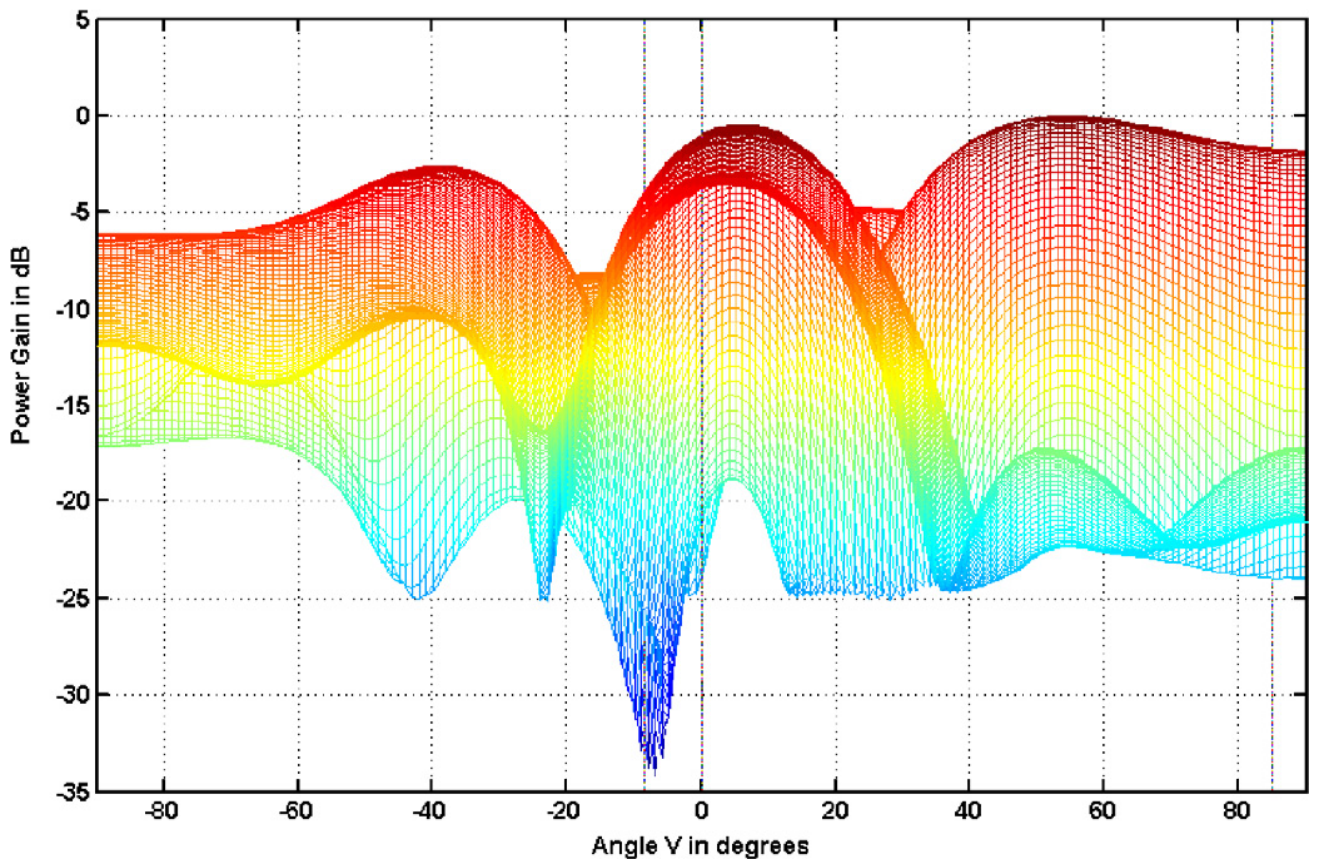

(b)

Fig. 6. Beam pattern with coherence using conventional method for Example 1.

rectangular pulse shape and $\mathrm{SNR}=0 \mathrm{~dB}$. The noise received by the UCA is spatially white. To facilitate plotting array beam patterns, we adopt the angle $(U, V)$ instead of $(\theta, \phi)$, where $\sin (U)=\sin (\theta) \sin (\phi)$ and $\sin (V)=\sin (\theta) \cos (\phi)$.

Example 1. Here, we set $M=9, L=7$, and $N=9$. Three signal sources are impinging on the array from angels $(\theta, \phi)=\left(55^{\circ}, 90^{\circ}\right),\left(10^{\circ}, 210^{\circ}\right)$, and $\left(85^{\circ}, 0^{\circ}\right)$, respectively. Assume that the desired signal is the first signal with $\left(55^{\circ}, 90^{\circ}\right)$ and gain $c_{1}=1$ and the others are the interferers with $c_{2}=c_{3}=0$. Fig. 3 plots the simulation results 


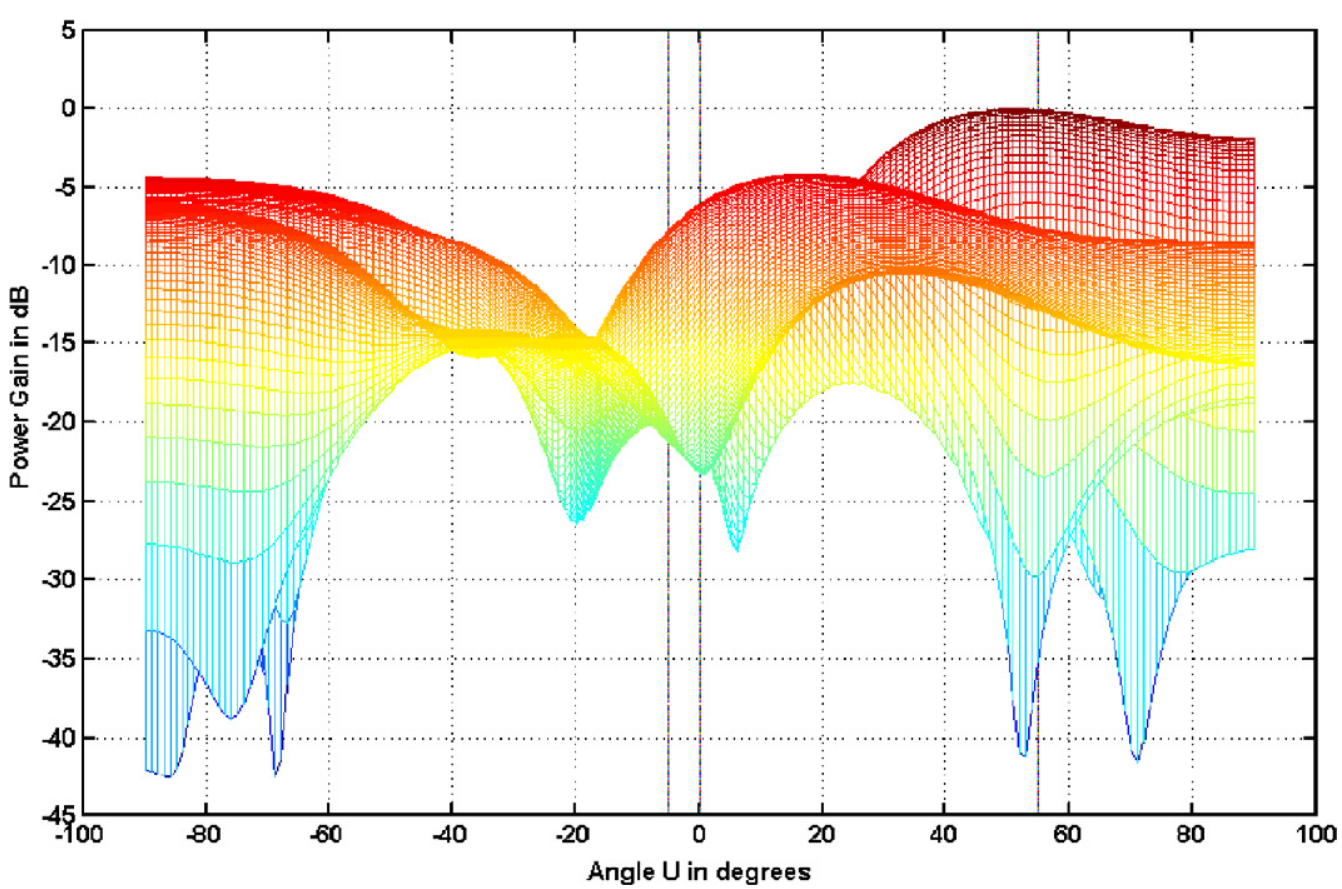

(a)

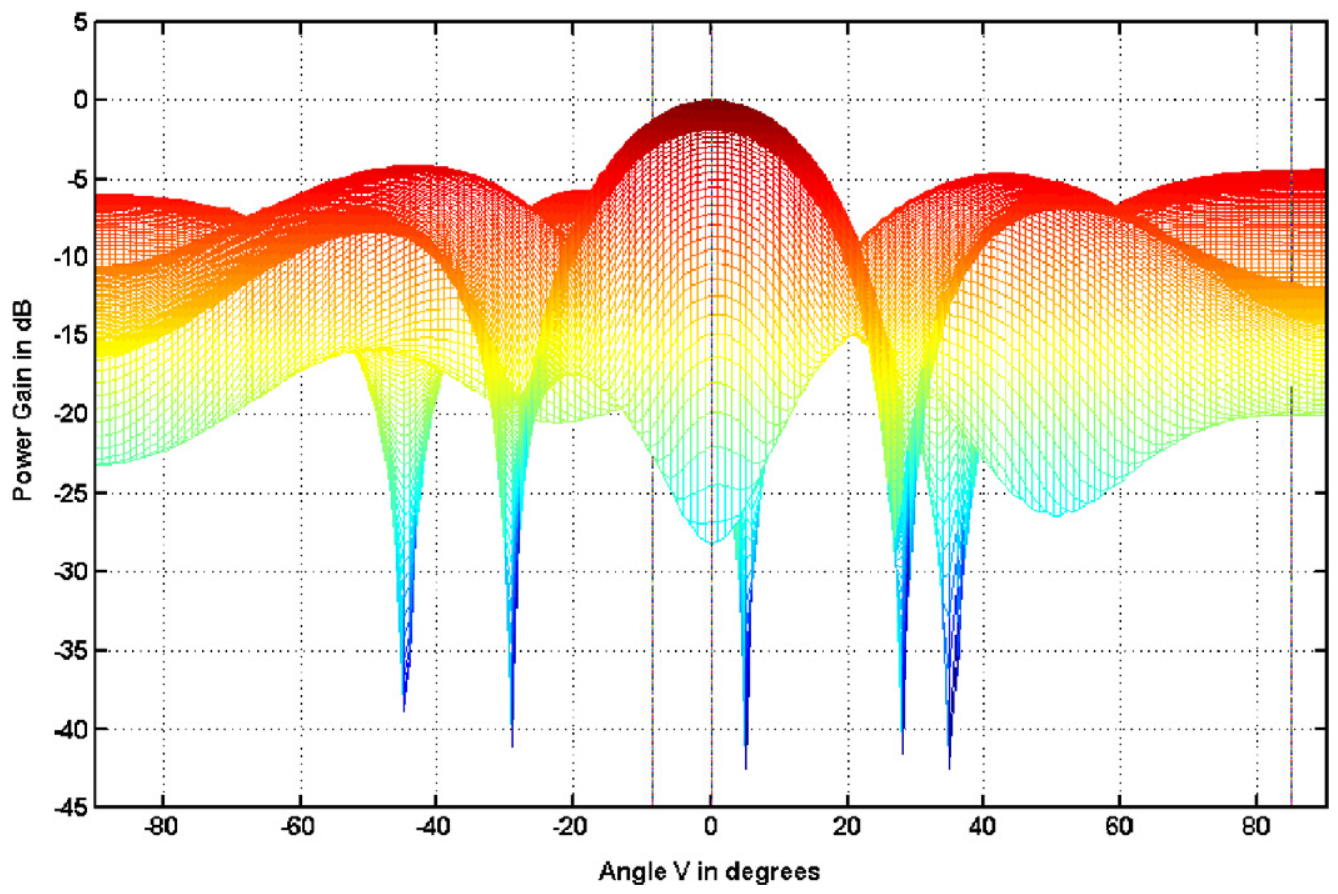

(b)

Fig. 7. Beam pattern using proposed method with averaging scheme for Example 1.

in terms of the array beam patterns using the proposed method of (26). For comparison, the results of using the conventional method of (7) are shown in Fig. 4. The array output signal-to-interference plus noise power ratio (SINR) obtained by using the proposed method and the conventional method are 8.3505 and $8.3227 \mathrm{~dB}$, respectively.

Next, let the interferer with $\left(85^{\circ}, 0^{\circ}\right)$ be coherent with the desired signal. Figs. 5 and 6 show the simulation results in terms of the array beam patterns. The output SINRs obtained by using the proposed method and the conventional 


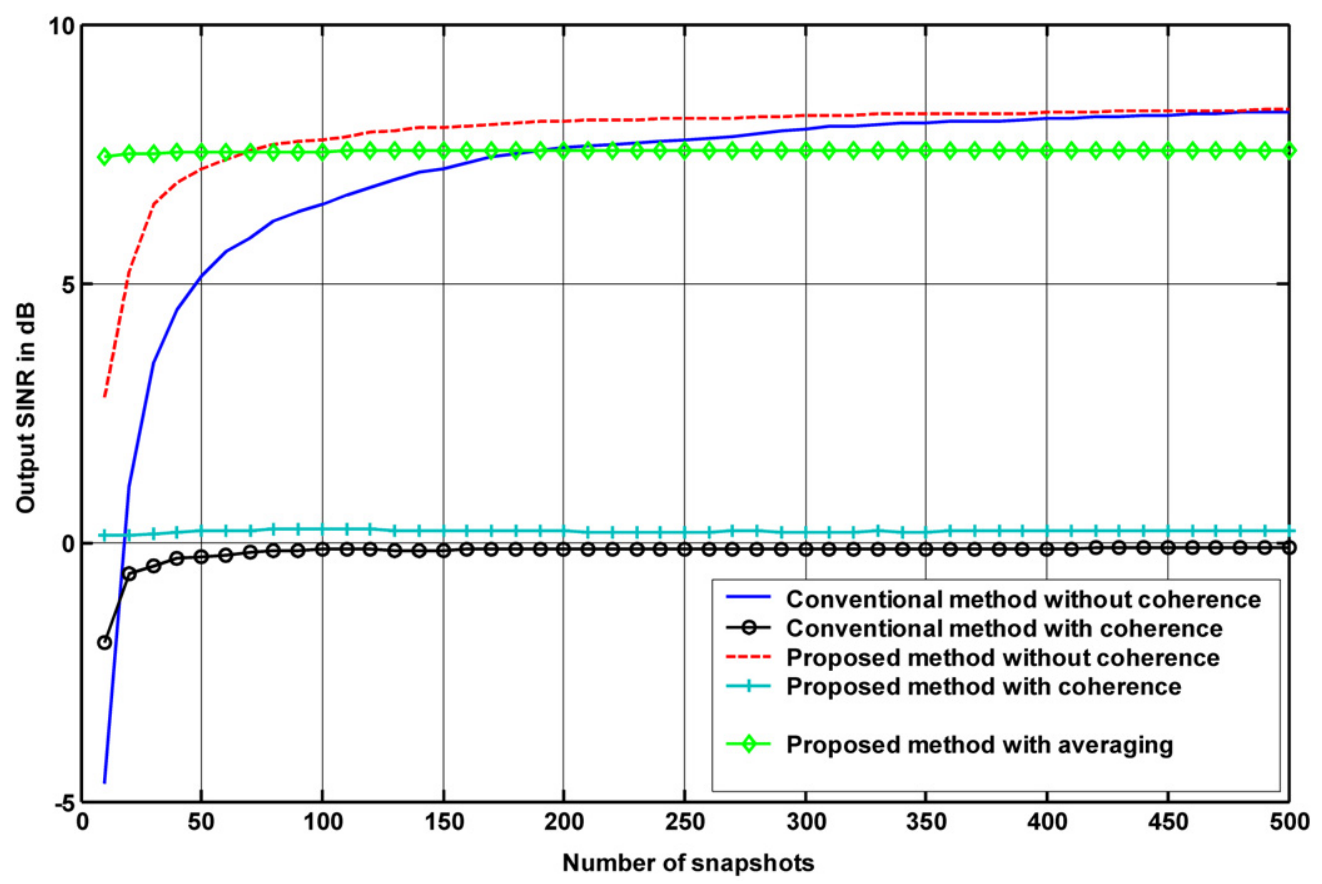

Fig. 8. The output SINR versus the number of snapshots for Example 1.

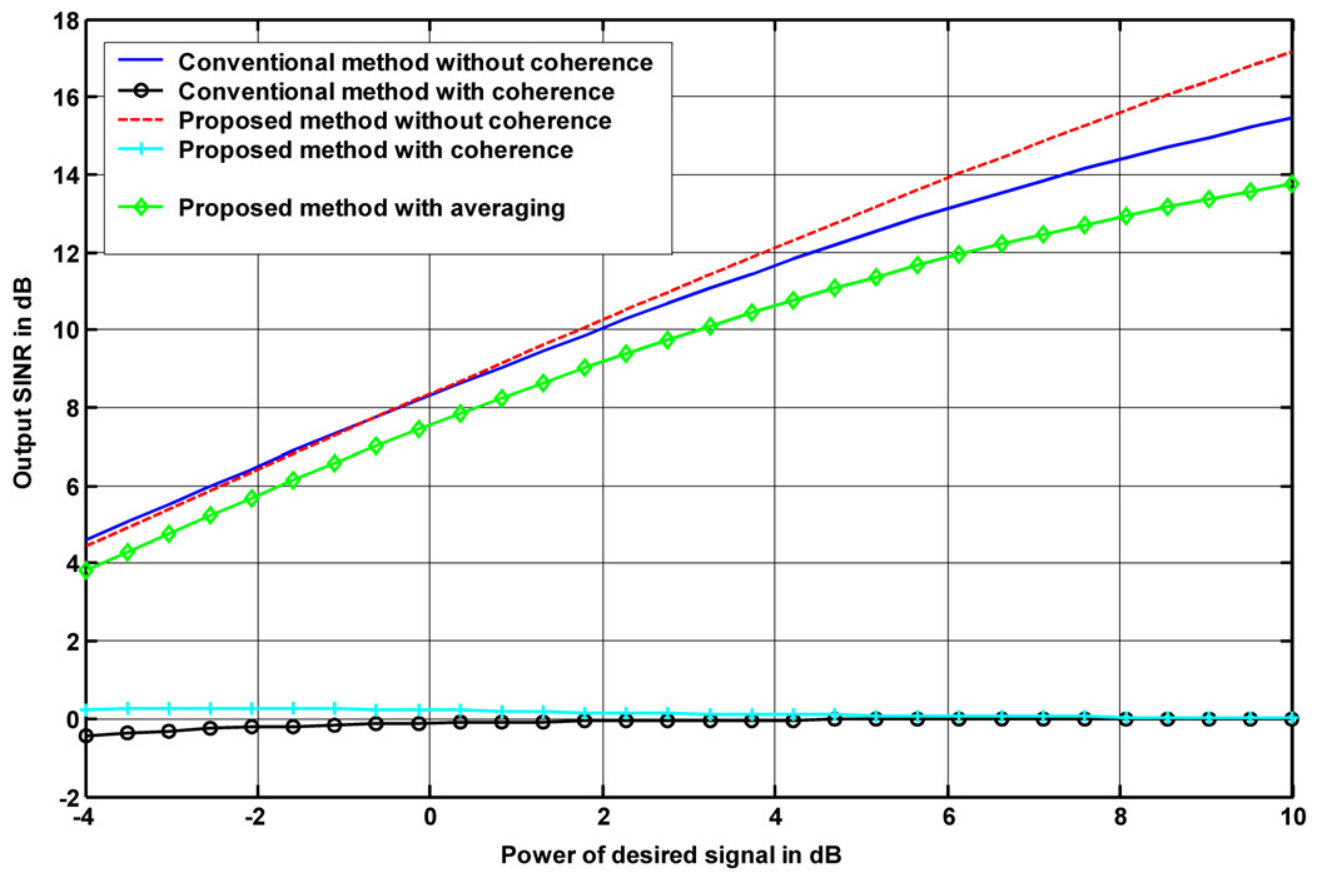

Fig. 9. The output SINR versus the power of desired signal for Example 1.

method are 0.2201 and $-0.1163 \mathrm{~dB}$, respectively. After applying the averaging scheme, the simulation results are depicted in Fig. 7. The output SINR of using the proposed method is 7.5595 dB. Fig. 8 plots the array output SINR versus the number of snapshots, while Fig. 9 depicts the array output SINR versus the power of the desired signal with the SNR of the interferers fixed at $0 \mathrm{~dB}$. The simulation results show that the proposed method works very well in the main-beam case. 


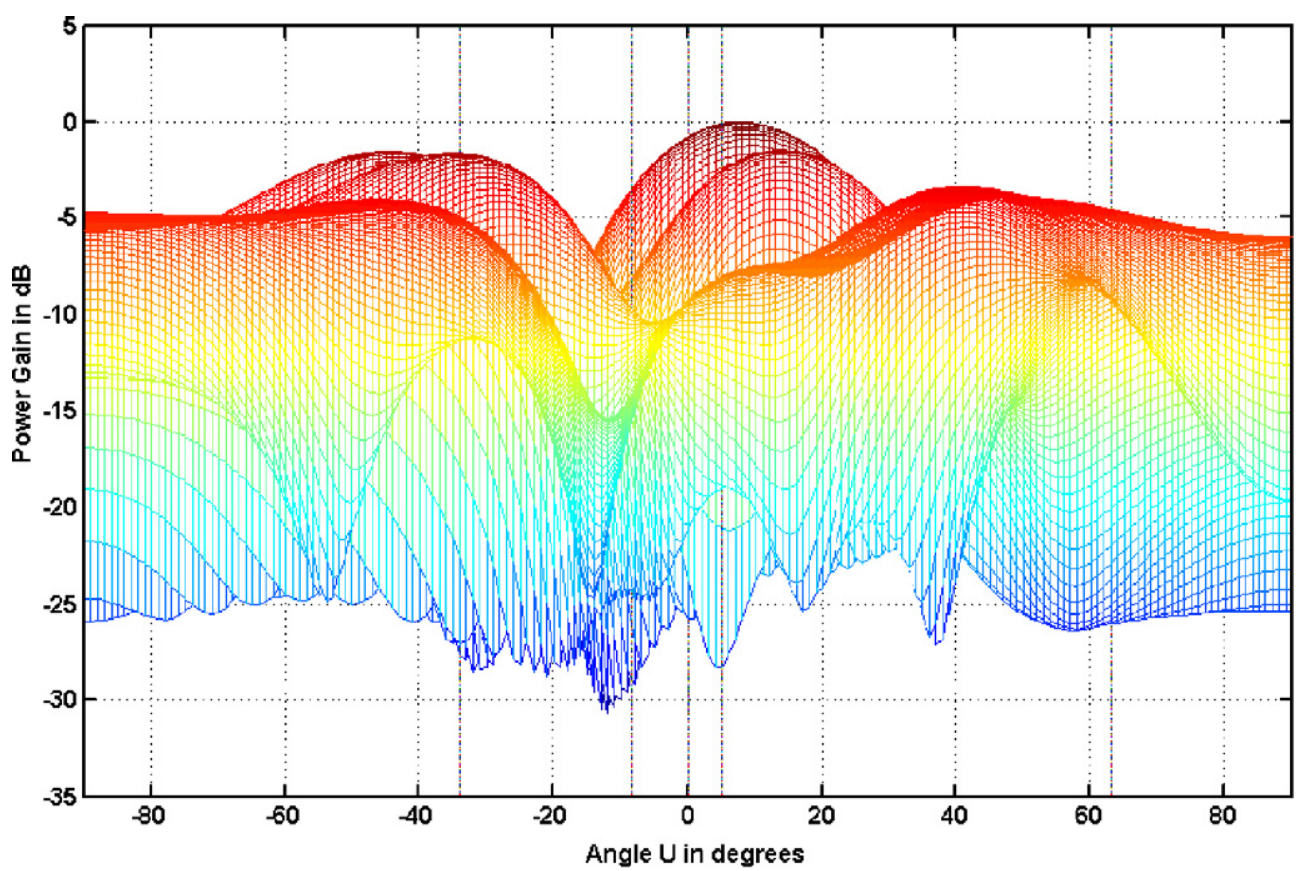

(a)

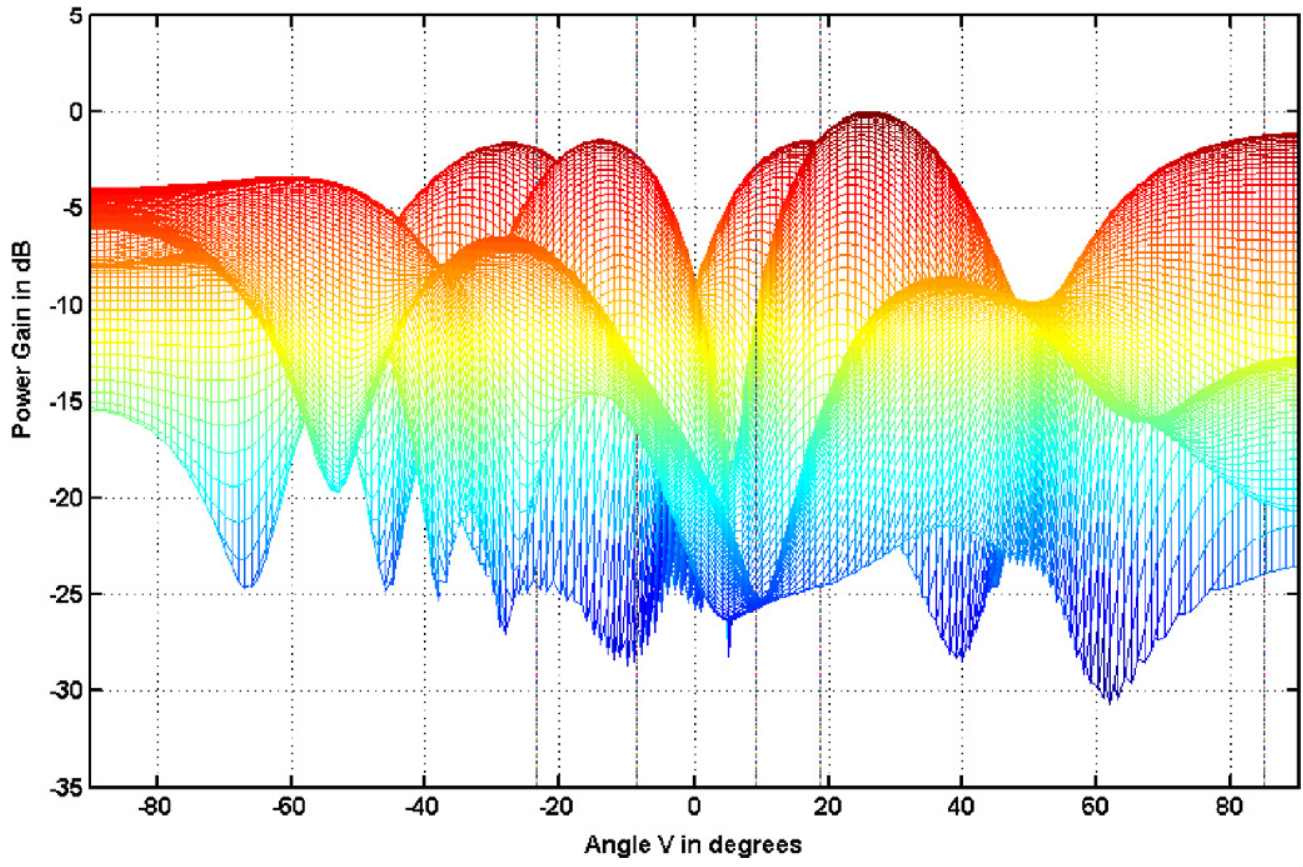

(b)

Fig. 10. Beam pattern without coherence using proposed method for Example 2.

Example 2. Here, we set $M=11, L=9$, and $N=11$. Five signal sources are impinging on the array from angels $(\theta, \phi)=\left(85^{\circ}, 0^{\circ}\right),\left(40^{\circ}, 300^{\circ}\right),\left(10^{\circ}, 150^{\circ}\right),\left(65^{\circ}, 80^{\circ}\right)$, and $\left(25^{\circ}, 200^{\circ}\right)$, respectively. Assume that the desired signals are the first three signals with $\left(85^{\circ}, 0^{\circ}\right),\left(40^{\circ}, 300^{\circ}\right),\left(10^{\circ}, 150^{\circ}\right)$ and gain $c_{1}=c_{2}=c_{3}=1$, and the others are the interferers with $c_{4}=c_{5}=0$. Figs. 10 and 11 plot the simulation results in terms of the array beam patterns using the proposed method of (26) and the conventional method of (7), respectively. The output SINRs of using the proposed method and the conventional method are 8.7331 and $8.5058 \mathrm{~dB}$, respectively. 


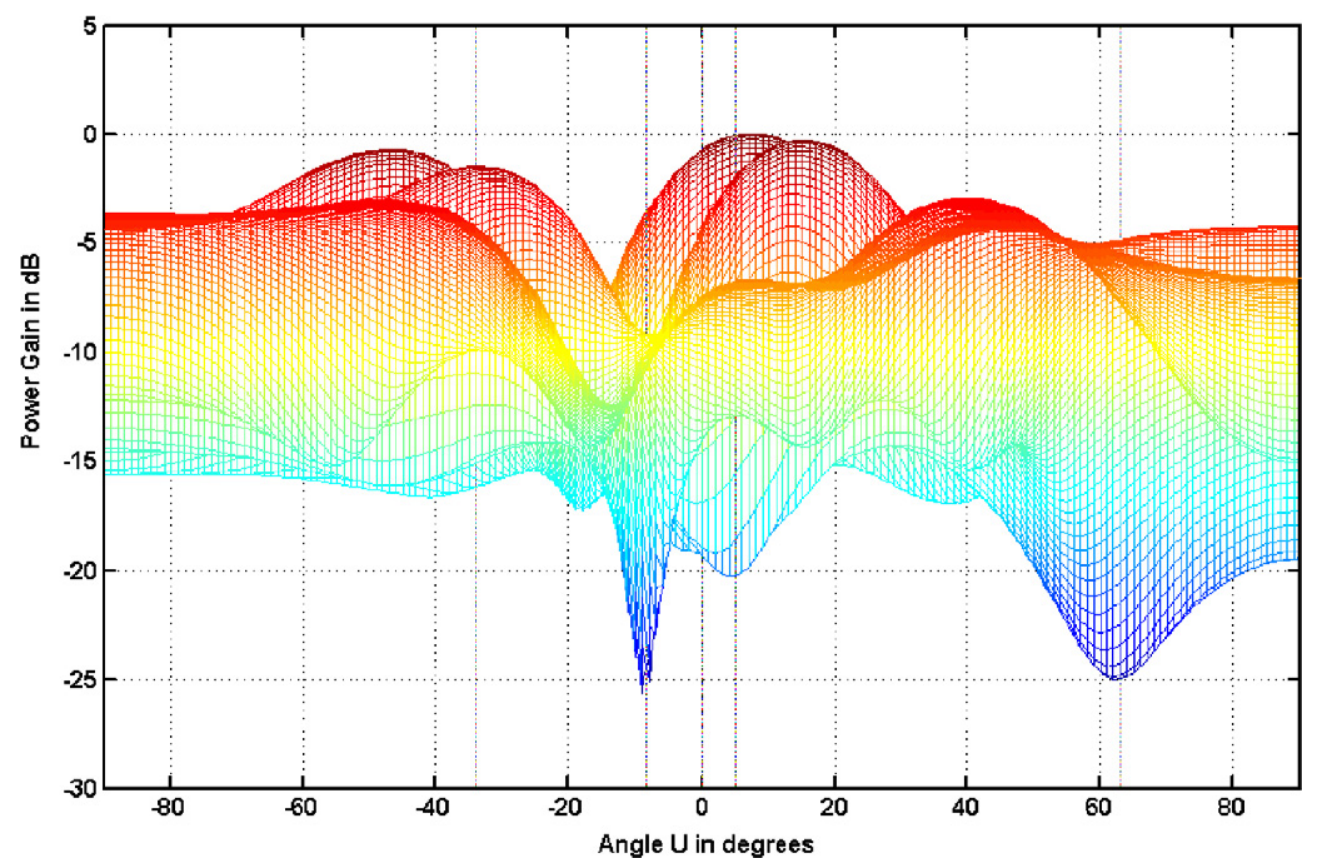

(a)

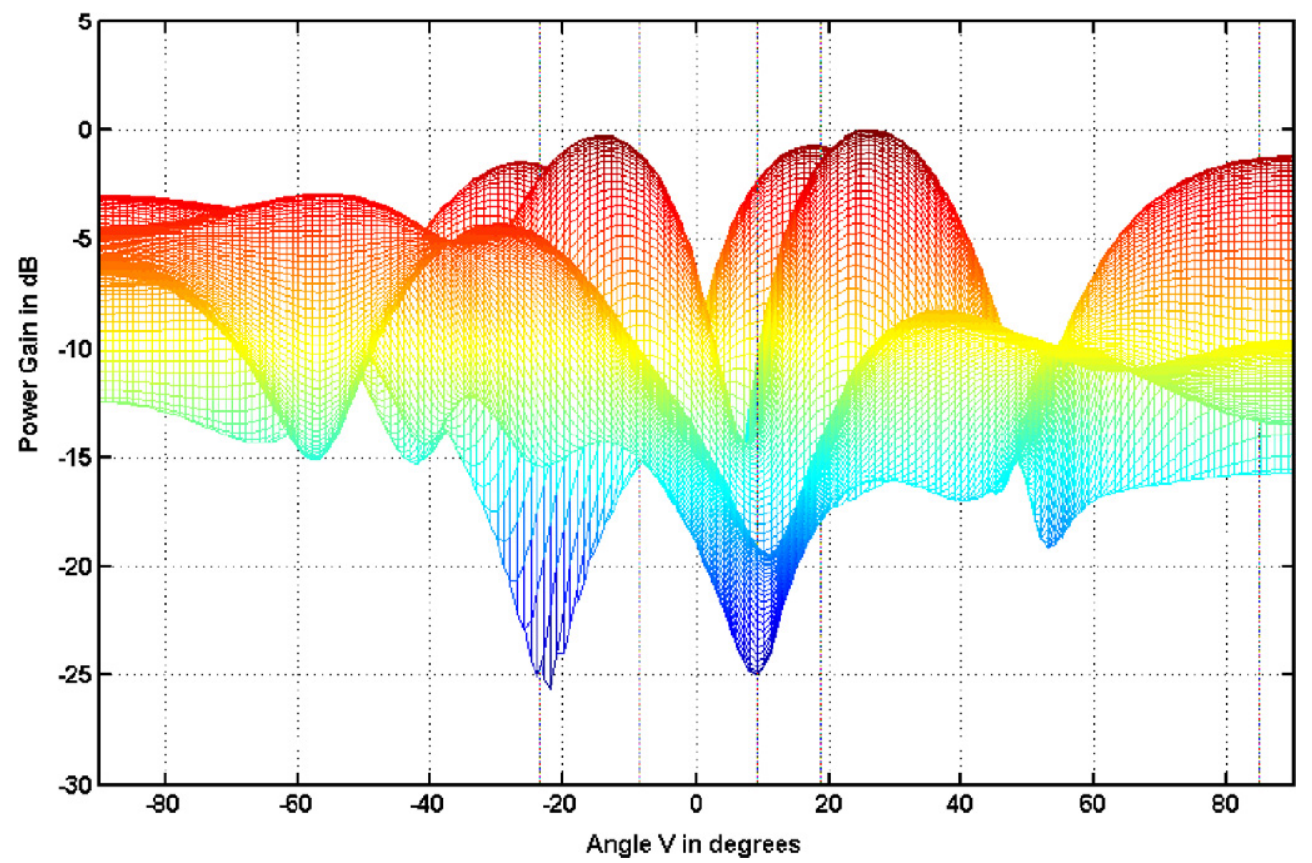

(b)

Fig. 11. Beam pattern without coherence using conventional method for Example 2.

Next, let the interferer of $\left(65^{\circ}, 80^{\circ}\right)$ be coherent with the desired signal of $\left(85^{\circ}, 0^{\circ}\right)$ and the interferer of $\left(25^{\circ}, 200^{\circ}\right)$ be coherent with the desired signal of $\left(40^{\circ}, 300^{\circ}\right)$. Figs. 12 and 13 show the simulation results in terms of the array beam patterns. The output SINRs obtained by using the proposed method and the conventional method are 1.5295 and $1.2875 \mathrm{~dB}$, respectively. After applying the averaging scheme, the simulation results are depicted in Fig. 14. The output SINR of using the proposed method is $8.5051 \mathrm{~dB}$. Finally, the array output SINR versus the number of snapshots is plotted in Fig. 15, while the array output SINR versus the power of the desired signals with the SNR 


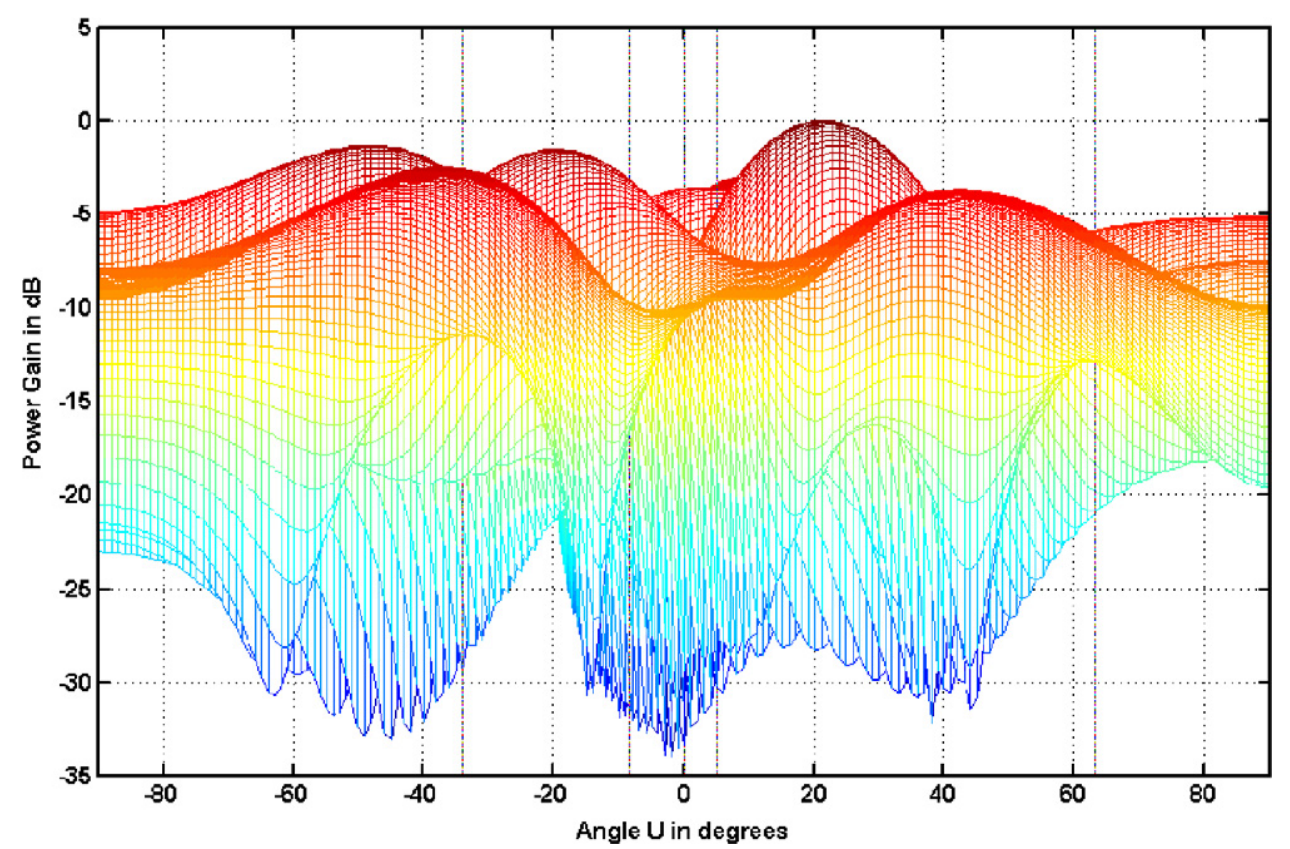

(a)

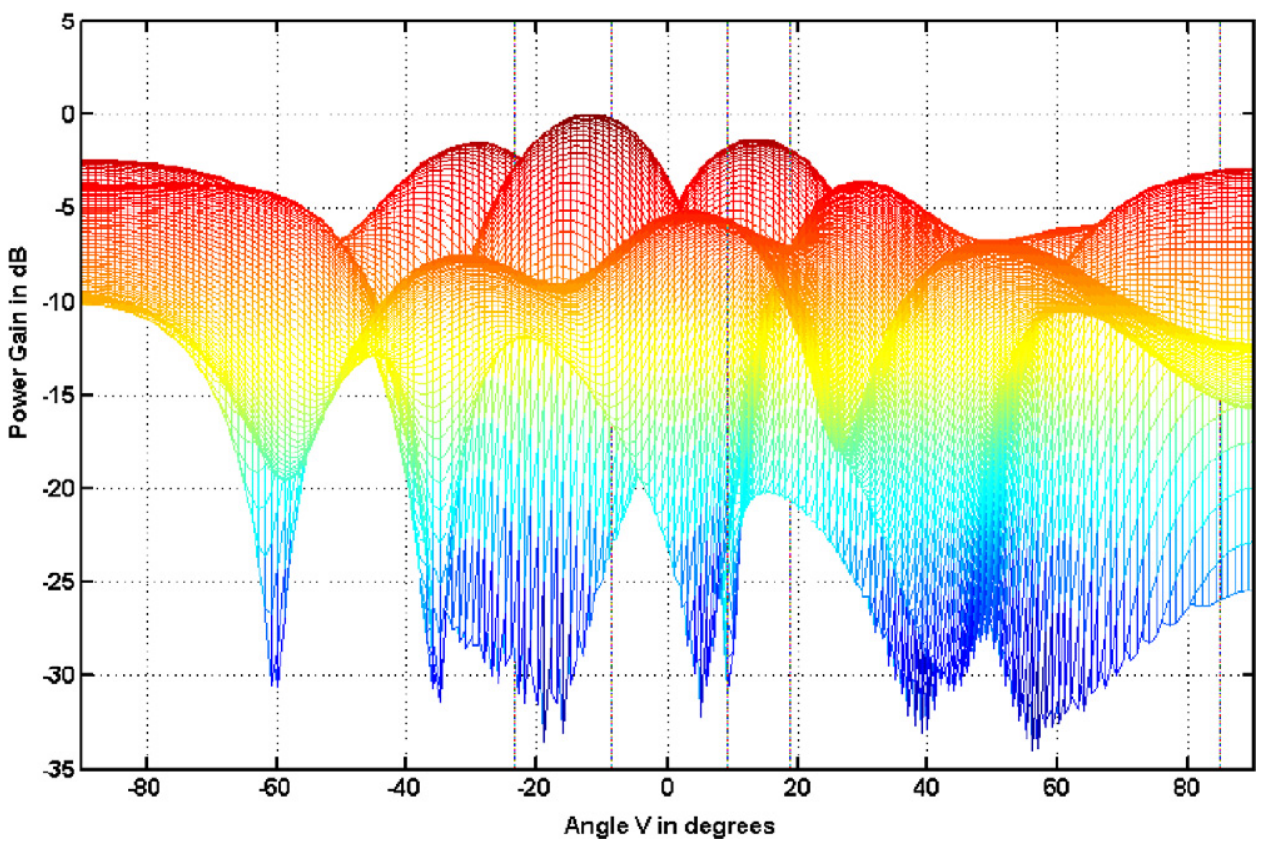

(b)

Fig. 12. Beam pattern with coherence using proposed method for Example 2.

of the interferers fixed at $0 \mathrm{~dB}$ is depicted in Fig. 16. The proposed method shows its effectiveness in the case with multiple-beam constraints.

\section{Conclusion}

This paper has presented an effective method for adaptive UCA beamforming under coherent interference. The proposed method utilizes a subarray beamforming in conjunction with an averaging scheme to effectively mitigate 


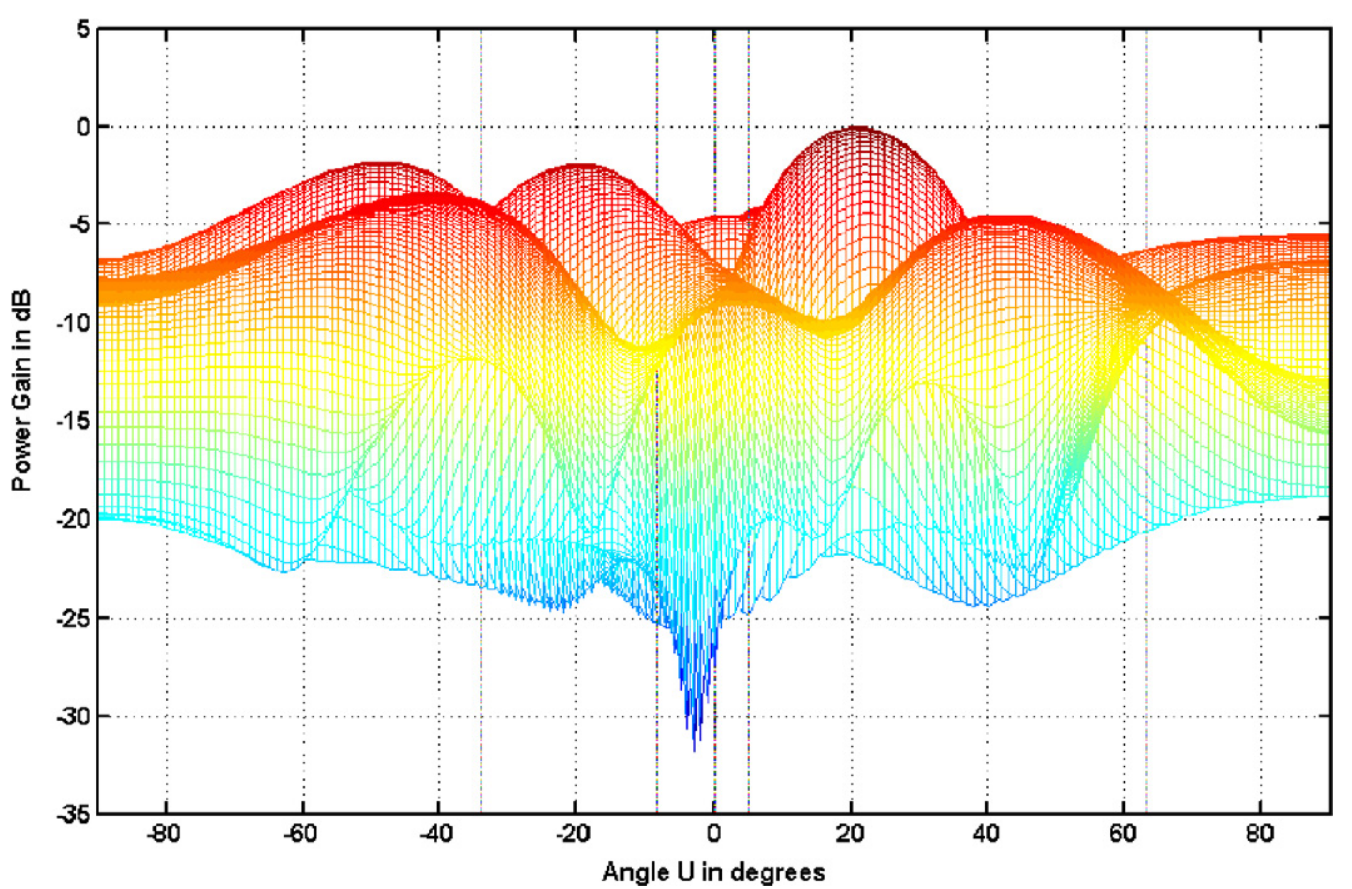

(a)

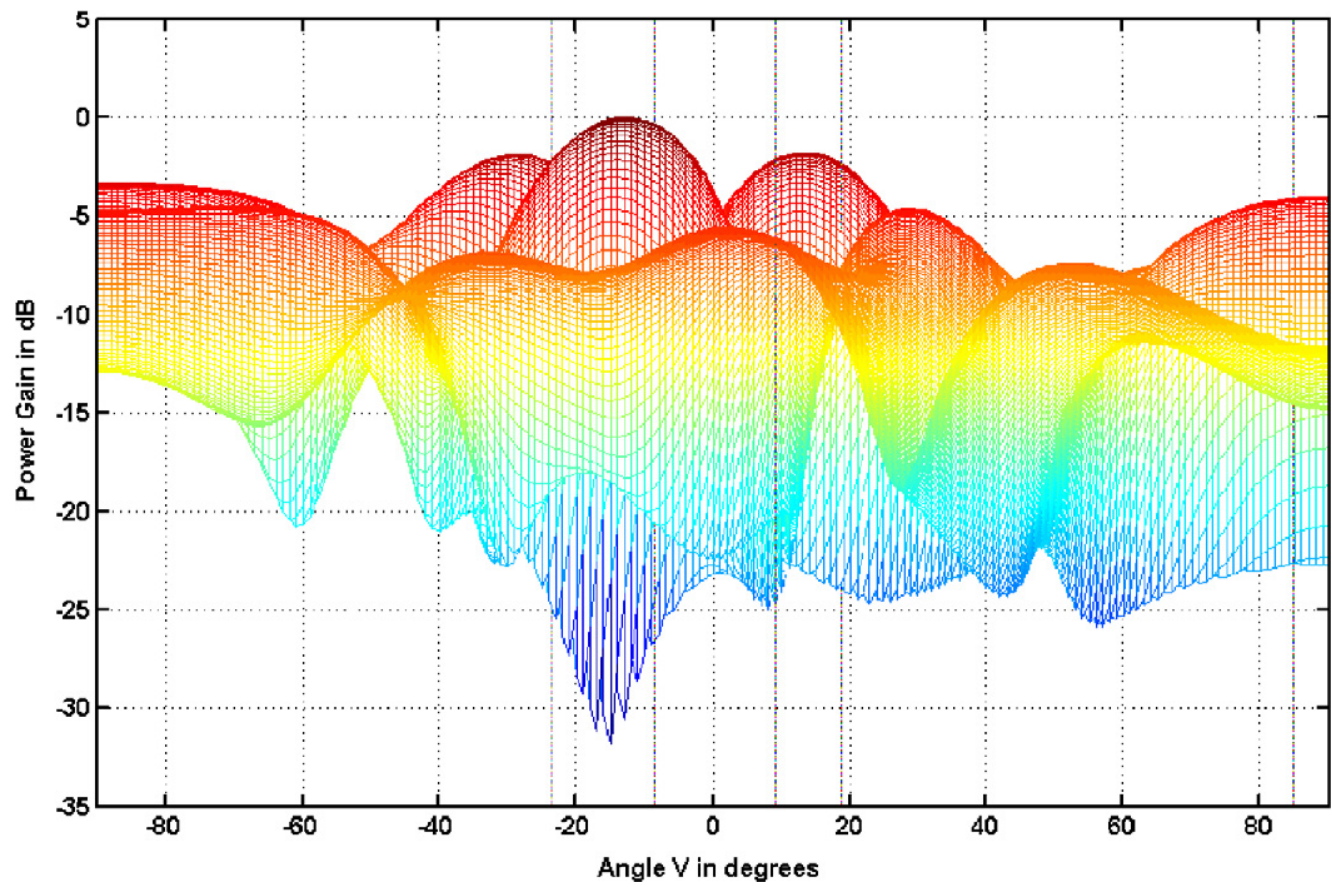

(b)

Fig. 13. Beam pattern with coherence using conventional method for Example 2.

the effect due to signal coherence. For processing array data, a UCA is partitioned into several overlapped subarrays. Each of the resulting subarrays is configured in a circular arc possessing the same radius as the original UCA. The overlapped subarrays are used for performing subarray beamforming and the averaging scheme. A procedure has been proposed to estimate the correlation matrix of the noise vector for facilitating a virtual beamforming which enhances the array performance. Theoretical analysis has shown the validity of the proposed method in dealing with the co- 


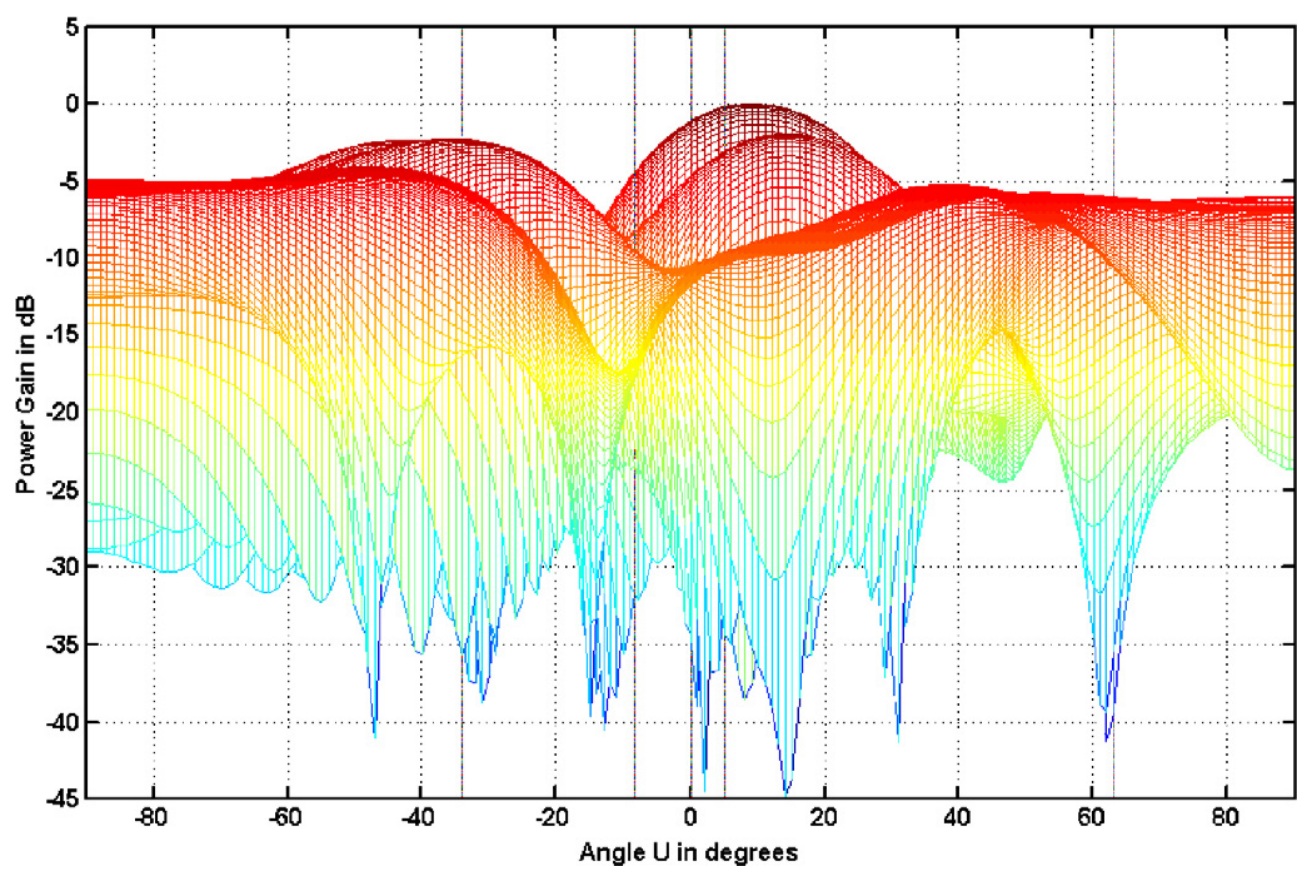

(a)

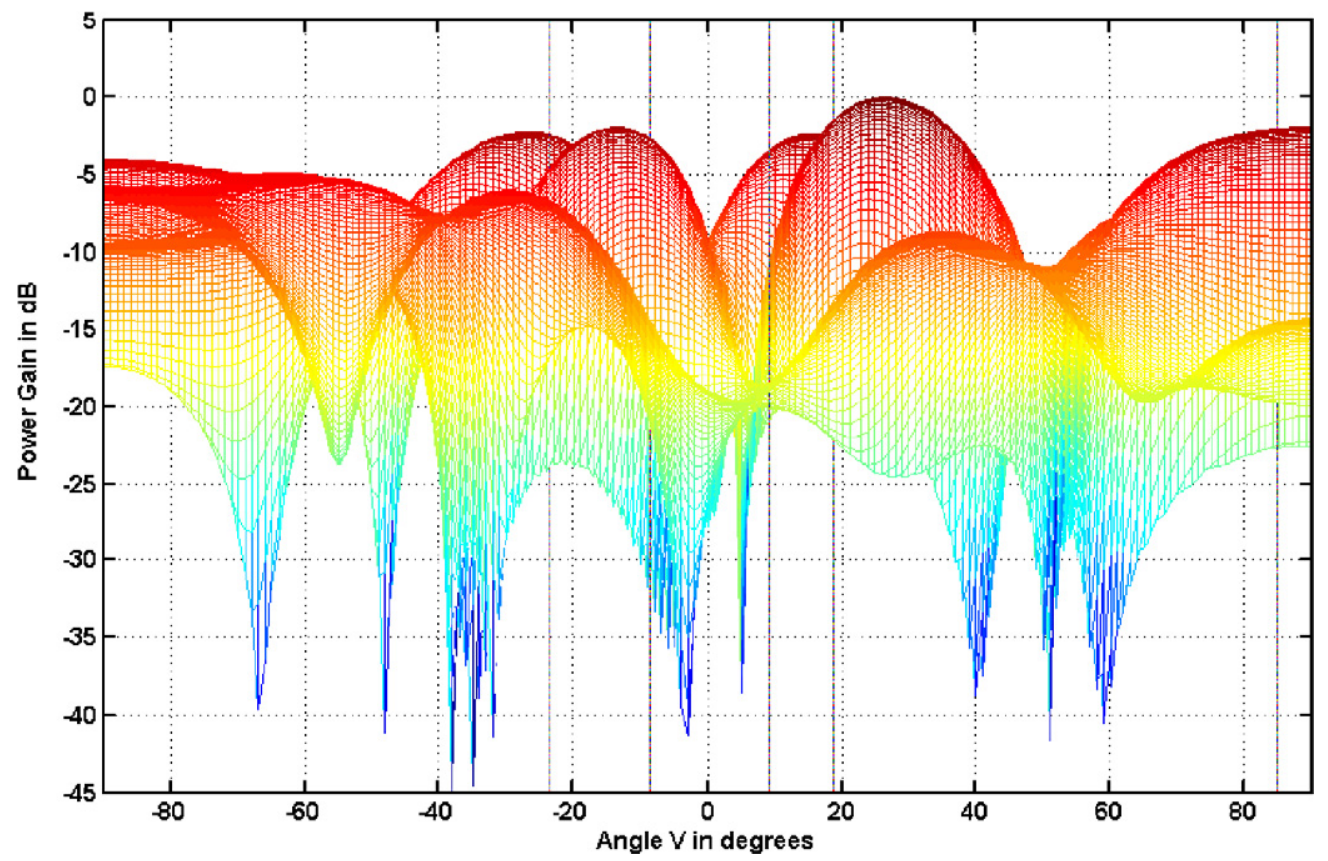

(b)

Fig. 14. Beam pattern using proposed method and averaging scheme for Example 2.

herent interference problem of a UCA. The effectiveness of the proposed method has been confirmed by simulation examples.

\section{Acknowledgment}

This work was supported by the National Science Council of Taiwan under Grant NSC95-2221-E002-186. 


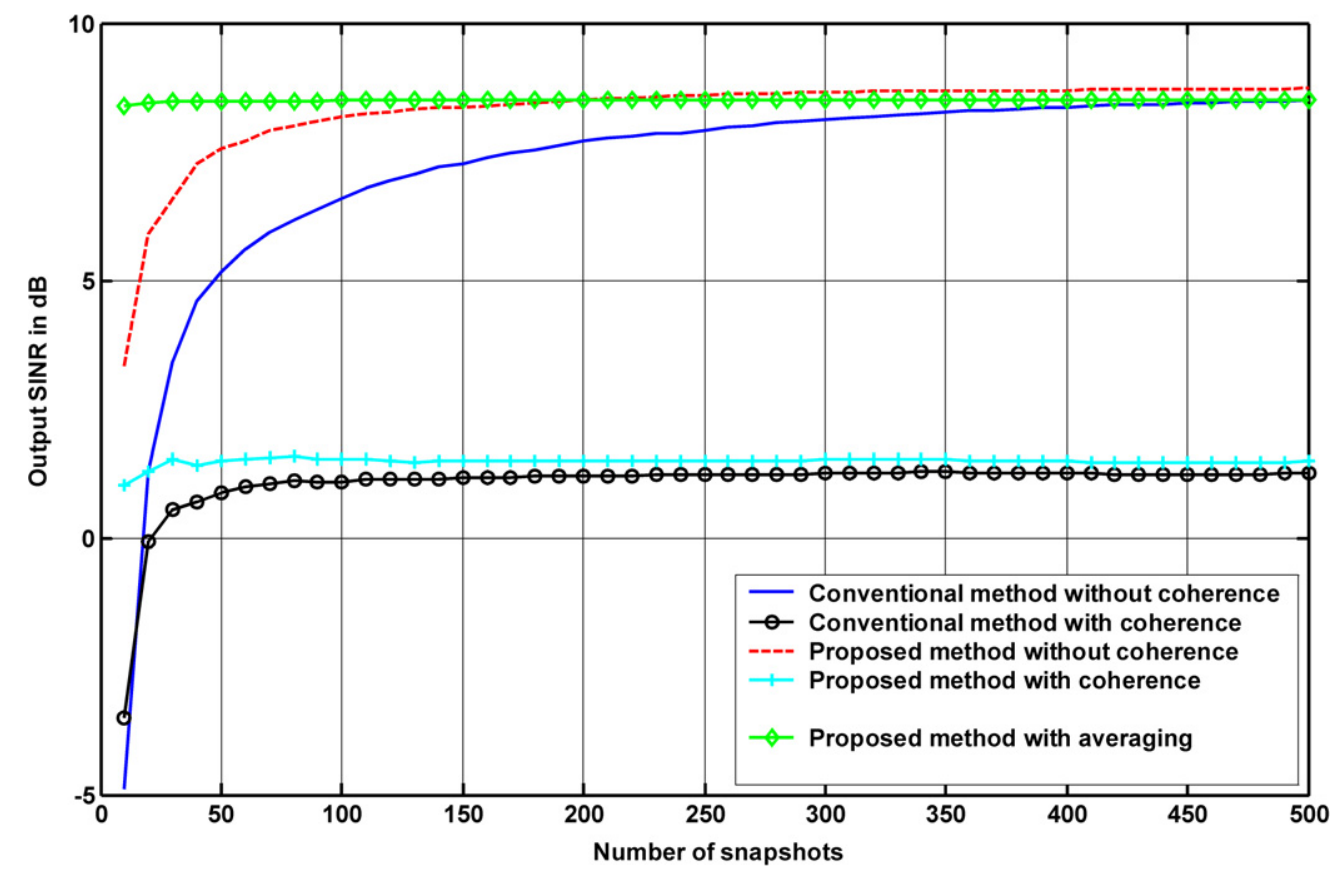

Fig. 15. The output SINR versus the number of snapshots for Example 2.

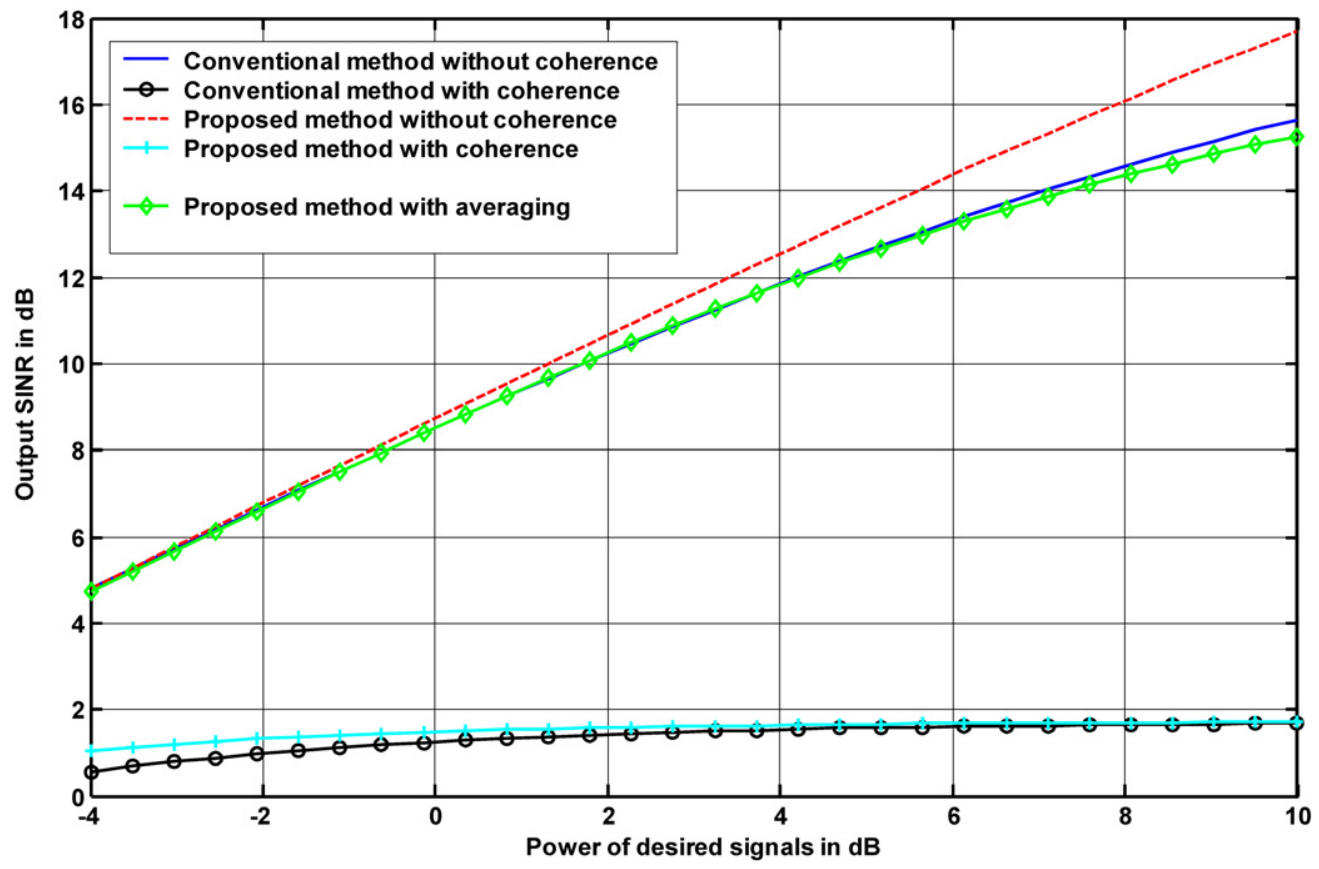

Fig. 16. The output SINR versus the power of desired signal for Example 2.

\section{References}

[1] M. Wax, J. Sheinvald, Direction finding of coherent signals via spatial smoothing for uniform circular arrays, IEEE Trans. Antenn. Propagat. 42 (1994) 613-620.

[2] K.M. Reddy, V.U. Reddy, Analysis of spatial smoothing with uniform circular arrays, IEEE Trans. Signal Process. 47 (6) (1999) $1726-1730$. 
[3] B.K. Lau, Y.H. Leung, Optimum beamformers for uniform circular arrays in a correlated signal environment, in: Proc. of IEEE International Conference on Acoustics, Speech and Signal Processing, vol. 5, Istanbul, Turkey, June 2000, pp. 3093-3096.

[4] J.-A. Tsai, B.D. Woerner, Adaptive beamforming of uniform circular arrays (UCA) for wireless CDMA system, in: Proc. of 35th Asilomar Conference on Signals, Systems and Computers, vol. 1, Pacific Grove, CA, November 2001, pp. 399-403.

[5] S. Durrani, M.E. Bialkowski, Effect of mutual coupling on the interference rejection capabilities of linear and circular arrays in CDMA systems, IEEE Trans. Antenn. Propagat. 52 (4) (2004) 1130-1134.

[6] T. Su, H. Ling, Adaptive beamforming in the presence of a mounting tower using genetic algorithms, IEEE Trans. Antenn. Propagat. 53 (6) (1986) 2011-2019.

[7] P. Ioannides, C.A. Balanis, Uniform circular and rectangular arrays for adaptive beamforming applications, IEEE Trans. Antenn. Wireless Propagat. Lett. 4 (2005) 351-354.

[8] S. Yan, Y. Ma, Robust supergain beamforming for circular array via second-order cone pregramming, Appl. Acoust. 66 (9) (2005) $1018-1032$.

[9] D.E.N. Davis, A transformation between the phasing techniques required for linear and circular arrays, Proc. IEE 112 (1965) $2041-2045$.

[10] I.D. Longstaff, P.E.K. Chow, D.E.N. Davis, Directional properties of circular arrays, Proc. IEE 114 (1967) 713-718.

[11] B. Sheleg, A matrix-fed circular array for continuous scanning, Proc. IEEE 56 (1968) 2016-2027.

[12] T. Rahim, D.E.N. Davis, Effect of directional elements on the directional response of circular antenna arrays, Proc. IEE 129 (Part H) (1982) $18-22$.

[13] M.A. Doron, E. Doron, A.J. Weiss, Coherent wide-band processing for arbitrary array geometry, IEEE Trans. Signal Process. 41 (1) (1993) $414-417$.

[14] A. Zeira, B. Friedlander, Interpolated array minimum variance beamforming for correlated interference rejection, in: Proc. of IEEE International Conference on Acoustics, Speech and Signal Processing, vol. 5, Atlanta, GA, June 1996, pp. 3165-3168.

[15] B.K. Lau, Y.H. Leung, Y. Liu, K.L. Teo, Transformations for nonideal uniform circular arrays operating in correlated signal environments, IEEE Trans. Signal Process. 54 (1) (2006) 34-48.

[16] T.J. Shan, T. Kailath, Adaptive beamforming for coherent signals and interference, IEEE Trans. Acoust. Speech Signal Process. 33 (1985) 527-536.

[17] B.L. Lim, S.K. Hui, Y.C. Lim, Bearing estimation of coherent sources by circular spatial modulation averaging (CSMA) technique, Electron. Lett. 26 (5) (1990) 343-345.

[18] N. Owsley, D. Abraham, Preprocessing for high resolution beamforming, in: Proc. of 23rd Asilomar Conference on Signals, Systems and Computers, November 1989, pp. 797-801.

[19] J.-H. Lee, F.-P. Tong, Efficient adaptive array beamforming under steering errors, in: Proc. of IEEE International Symposium on Antennas and Propagation, Chicago, IL, July 1992, pp. 1015-1018.

[20] J.A. Nuttall, P. Willett, Adaptive-adaptive subarray narrowband beamforming, in: Proc. of IEEE International Conference on Acoustics, Speech and Signal Processing, Minneapolis, MN, April 1993, pp. 305-308.

[21] M. Wax, T. Kailath, Detection of signals by information theoretic criteria, IEEE Trans. Acoust. Speech Signal Process. 33 (1985) $387-392$.

[22] R.A. Horn, C.R. Johnson, Matrix Analysis, Cambridge Univ. Press, Cambridge, 1985.

Ju-Hong Lee was born in I-Lan, Taiwan, on December 7, 1952. He received the B.S. degree from the National Cheng-Kung University, Tainan, Taiwan, in 1975, the M.S. degree from the National Taiwan University, Taipei, Taiwan, in 1977, and the Ph.D. degree from Rensselaer Polytechnic Institute, Troy, NY, USA, in 1984, all in electrical engineering.

From September 1980 to July 1984, he was a Research Assistant and was involved in research on multidimensional recursive digital filtering in the Department of Electrical, Computer, and Systems Engineering at Rensselaer Polytechnic Institute. From August 1984 to July 1986, he was a Visiting Associate Professor and later in August 1986 became an Associate Professor in the Department of Electrical Engineering, National Taiwan University (NTU). Since August 1989, he has been a Professor at the same university. He was appointed Visiting Professor in the Department of Computer Science and Electrical Engineering, University of Maryland, Baltimore, USA, during a sabbatical leave in 1996. His current research interests include multidimensional digital signal processing, image processing, detection and estimation theory, analysis and processing of joint vibration signals for the diagnosis of cartilage pathology, and adaptive signal processing and its applications in communications.

Dr. Lee received Outstanding Research Awards from the National Science Council (NSC) in the academic years of 1988, 1989, and 1991-1994, and Distinguished Research Awards from the NSC in the academic years of 1998-2004, and the NSC Research Fellowship in the academic years of 2005-2008. In August 2006, he was appointed NTU's Tenured Distinguished Professor.

Shuo-Wei Hsiao was born in Taipei County, Taiwan, on October 15, 1981. He received the B.S. degree in electrical engineering from the National Chung-Cheng University, Chia-Yi, Taiwan, in 2004, and the M.S. degree in communication engineering from the National Taiwan University, Taipei, Taiwan, in 2006. His current research interests include statistical signal processing and its applications. 